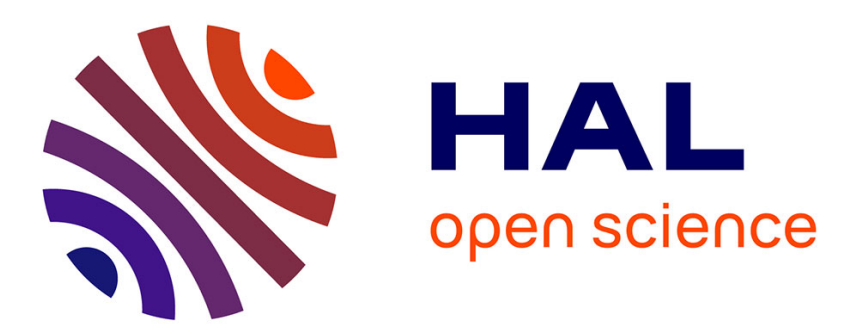

\title{
Moonlet Induced Wakes in Planetary Rings: Analytical Model Including Eccentric Orbits of Moon and Ring Particles
}

\author{
M. Seiss, F. Spahn, Jürgen Schmidt
}

\section{- To cite this version:}

M. Seiss, F. Spahn, Jürgen Schmidt. Moonlet Induced Wakes in Planetary Rings: Analytical Model Including Eccentric Orbits of Moon and Ring Particles. Icarus, 2010, 210 (1), pp.298. 10.1016/j.icarus.2010.06.013 . hal-00683832

\section{HAL Id: hal-00683832 https://hal.science/hal-00683832}

Submitted on 30 Mar 2012

HAL is a multi-disciplinary open access archive for the deposit and dissemination of scientific research documents, whether they are published or not. The documents may come from teaching and research institutions in France or abroad, or from public or private research centers.
L'archive ouverte pluridisciplinaire HAL, est destinée au dépôt et à la diffusion de documents scientifiques de niveau recherche, publiés ou non, émanant des établissements d'enseignement et de recherche français ou étrangers, des laboratoires publics ou privés. 


\section{Accepted Manuscript}

Moonlet Induced Wakes in Planetary Rings: Analytical Model Including Eccentric Orbits of Moon and Ring Particles

M. Seiß, F. Spahn, Jürgen Schmidt

PII:

S0019-1035(10)00235-6

DOI:

10.1016/j.icarus.2010.06.013

Reference:

YICAR 9465

To appear in:

Icarus

Received Date: 3 December 2008

Revised Date: $\quad 3$ June 2010

Accepted Date: 11 June 2010

Please cite this article as: Seiß, M., Spahn, F., Schmidt, J., Moonlet Induced Wakes in Planetary Rings: Analytical Model Including Eccentric Orbits of Moon and Ring Particles, Icarus (2010), doi: 10.1016/j.icarus.2010.06.013

This is a PDF file of an unedited manuscript that has been accepted for publication. As a service to our customers we are providing this early version of the manuscript. The manuscript will undergo copyediting, typesetting, and review of the resulting proof before it is published in its final form. Please note that during the production process errors may be discovered which could affect the content, and all legal disclaimers that apply to the journal pertain. 


\title{
Moonlet Induced Wakes in Planetary Rings: Analytical Model Including Eccentric Orbits of Moon and Ring Particles
}

\author{
M. Seiß ${ }^{1}$, F. Spahn ${ }^{1}$ and Jürgen Schmidt ${ }^{1}$
}

Nonlinear Dynamics Group, Institute of Physics and Astronomy, University of Potsdam, 14476, Golm, Germany

Received accepted

${ }^{1}$ Nonlinear Dynamics Group, Institute of Physics and Astronomy, University of Potsdam, 14476, Golm, Germany. 


\begin{abstract}
Saturn's rings host two known moons, Pan and Daphnis, which are massive enough to clear circumferential gaps in the ring around their orbits. Both moons create wake patterns at the gap edges by gravitational deflection of the ring material (Cuzzi and Scargle (1985), Showalter et al. (1986)). New Cassini observations revealed that these wavy edges deviate from the sinusoidal waveform, which one would expect from a theory that assumes a circular orbit of the perturbing moon and neglects particle interactions. Resonant perturbations of the edges by moons outside the ring system, as well as an eccentric orbit of the embedded moon, may partly explain this behavior (Porco et al. (2005); Tiscareno et al. (2005); Weiss et al. $(2005,2009))$. Here we present an extended non-collisional streamline model which accounts for both effects. We describe the resulting variations of the density structure and the modification of the nonlinearity parameter $q$. Furthermore, an estimate is given for the applicability of the model. We use the streamwire model introduced by Stewart (1991) to plot the perturbed ring density at the gap edges.

We apply our model to the Keeler gap edges undulated by Daphnis and to a faint ringlet in the Encke gap close to the orbit of Pan. The modulations of the latter ringlet, induced by the perturbations of Pan (Burns et al. (2005)), can be well described by our analytical model. Our analysis yields a Hill radius of Pan of $17.5 \mathrm{~km}$, which is $9 \%$ smaller than the value presented by Porco et al. (2005), but fits well to the radial semi-axis of Pan of $17.4 \mathrm{~km}$. This supports the idea that Pan has filled its Hill sphere with accreted material (Porco et al. (2007)). A numerical solution of a streamline is used to estimate the parameters of the Daphnis Keeler gap system, since the close proximity of the gap edge to the moon induces strong perturbations, not allowing an application of the analytic
\end{abstract}


streamline model. We obtain a Hill radius of $5.1 \mathrm{~km}$ for Daphnis, an inner edge variation of $8 \mathrm{~km}$, and an eccentricity for Daphnis of $1.5 \cdot 10^{-5}$. The latter two quantities deviate by a factor of two from values gained by direct observations (Jacobson et al. (2008); Tiscareno et al. (2005)), which might be attributed to the neglect of particle interactions and vertical motion in our model.

Subject headings: PLANETARY RINGS; SATURN, RINGS; SATURN, SATELLITES; SATELLITES, DYNAMICS; RESONANCES, RINGS; DISKS 


\section{Introduction}

Saturn's dense rings consist mainly of icy particles with sizes ranging from centimeters up to several meters (e.g. Zebker et al. (1985)). Recent observations by the spacecraft Cassini have shown that a large number of small moons (moonlets) are embedded in Saturn's dense rings being much larger than the usual ring particles (Tiscareno et al. (2006); Sremčević et al. (2007); Tiscareno et al. (2008)). The gravity of the moons induces characteristic density structures in their vicinity. Depending on their gravitational action on the ring material one can classify the moonlets in two size classes.

On the one hand there exist large moonlets with diameters roughly larger than 1 $\mathrm{km}$, which are able to clear a almost empty gap around their orbit due to their gravity (Henon (1981); Lissauer et al. (1981); Petit and Henon (1988); Spahn and Wiebicke (1989)). Particles close to the orbit of the moon are scattered efficiently out of the gap region while particles at larger radial distance are just deflected moderately. In the latter case the change of the particles' semimajor axes due to the moon gravity is negligible, while their eccentricities change significantly. This systematic deflection results in a sinusoidal spatial oscillation of the particles relative to the moon, leading to a modulation of the gap edge and a wavy appearance of the ring surface density close to the edge (Cuzzi and Scargle (1985); Showalter et al. (1986)). This pattern is known as moonlet induced wakes and should not be confused with the wakes caused by the self gravity of the ring particles (Salo $(1992,1995)$; Colwell et al. (2006); Thomson et al. (2007)). If necessary, the terms moonlet wakes and self gravity wakes will be used to distinguish both ring phenomena. The particles orbiting closest to the moonlet orbit move on horseshoe orbits which can only become unstable if other perturbations are taken into account. Thus, a ringlet can exist on the orbit of the moon (Spahn and Wiebicke (1989)). The observation of wakes at the edges of the Encke gap and a central ringlet inside this gap indicated the existence of a moon (Showalter et al. 
(1986); Spahn and Wiebicke (1989); Spahn and Sponholz (1989)). Finally, Showalter (1991) discovered the moon Pan in Voyager images. Fourteen years later a second moon, called Daphnis, was identified in the Keeler gap by the Cassini imaging team (Porco (2005)).

In contrast to the action of larger embedded moonlets the gravity of smaller moonlets $(\lesssim 1 \mathrm{~km})$ is not sufficient to create complete gaps in the ring going around the whole circumference. Diffusion of the ring material due to particle interactions (collisions, self gravity) smoothes out the induced density modulation before it can propagate around the circumference. Thus, a static S-shaped structure, called propeller, appears as a consequence of the interplay of moon gravity and particle collisions (Spahn and Sremčević (2000); Sremčević et al. (2002); Seiß et al. (2005)), which could be detected by the spacecraft Cassini (Tiscareno et al. (2006); Sremčević et al. (2007); Tiscareno et al. (2008)).

The present study concentrates on the formation of wakes induced by embedded moonlets. Showalter et al. (1986) developed a non-collisional streamline model to investigate the Pan wakes. The following restrictions were made by their model:

(i) particles move on circular orbits before they are scattered by the moon,

(ii) they have no additional (thermal) velocities relative to each other,

(iii) they do not interact with each other,

(iv) the moon is assumed on a circular orbit

(v) the moonlet wakes damp completely during one synodic period and

(vi) the motion of moon and ring particles is restricted to Saturn's equatorial plane.

Several extensions to the model proposed by Showalter et al. (1986) were made in the following years. Stewart (1991) included the effect of thermal velocities of particles, still 
neglecting particle collisions. Spahn et al. (1994) included the effect of multiple scattering by the moon, because the wakes are not always damped out after one synodic period (Horn et al. (1996)). Furthermore, the damping of the wakes has been modelled numerically by Borderies et al. (1989) using a hydrodynamic model and by Hertzsch et al. (1997) and Lewis and Stewart (2000) using N-body simulations.

Cassini observations showed that the perturbed edges of the Encke and Keeler gap deviate from the strict sinusoidal form (Porco et al. (2005); Tiscareno et al. (2005)) expected from the basic analytical model (Cuzzi and Scargle (1985); Showalter et al. (1986)). Resonances perturbing the gap edges, as well as a gap moon on an eccentric orbit, were proposed and could at least explain the observations partly (Weiss et al. (2005, 2009)). Moreover, a small but perhaps significant eccentricity of Pan of $1.4 \cdot 10^{-5}$ was determined (Jacobson et al. (2008)) which was earlier suggested by models of the central Encke gap ringlet (Spahn et al. (1992b)). This motivated a further development of the analytical models of Showalter et al. (1986) and Stewart (1991) accounting for particles and moonlets on eccentric orbits which is presented in this paper.

No model has so far considered the effect of non-planar motions of moon and particles on the edge waves and for simplicity we also neglect this effect. However, this might turn out to be important to understand the vertical variations of the edge waves induced by Daphnis, which become apparent from shadows of the wakes in images (Weiss et al. (2009); Hahn (2007)).

The article is organized as follows: In the next section the analytical solution by Moons et al. (1988), accounting for initially eccentric orbits of moonlet and ring particles, is extended to the fourth order of the inverse impact parameter and by an additional parameter (azimuthal guiding center position at $t=0$, see next section) which has not been considered so far. This solution can be used to model perturbed gap edges if the distance 
to the moon is sufficiently large. The limit of applicability of the analytical model is quantified. Furthermore, the analytical streamline solution is used to derive the perturbed ring density in the wake region (section 3) applying the method outlined by Borderies et al. (1982) and Showalter et al. (1986), but accounting also for initially non-circular orbits of moon and ring particles. This solution is suitable to model the optical depth profiles gained from occultation scans provided by the Cassini instruments (UVIS, VIMS, RSS). The density model derived in section 3 cannot describe the wakes directly at the gap edges. Thus, in section 4 we used an extension of the Stewart (1991) streamwire model, in order to compute the density at the edges using the anlytical streamline solution. In section 5 we use a numerical solution for streamlines in order to fit the gap edges. In this way we are able to judge the applicability of the analytical model depending on the eccentricities and the impact parameter. We summarize and discuss our results in section 6 .

\section{Streamline Model}

In order to investigate the influence of the moon gravity on the ring density one needs to calculate the deflection of the particles. Therefore, the equation of motion has to be solved, describing an encounter of two particles moving around a central body. The solution for large impact parameters is derived as proposed by Henon and Petit (1986). Later, this solution is used in order to calculate the ring density in the wake regions.

\subsection{Equations of Motion}

In general we have a system of three massive bodies moving under their mutual gravitational interaction (general three body problem). Several assumptions can be made in order to get a simpler set of equations. First of all, the mass of the central body is much 
larger than the mass of the moon and the ring particles. This also means that the gravity field of the central body dominates the motion of particle and moon most of the time. Only close encounters of moon and ring particle lead to noticeable deflections. Furthermore, the mass of the moon is much larger than the mass of a ring particle. Finally, a planar motion of all three bodies (central body, moon, ring particle) is assumed restricting the particle motion to two dimensions. With these simplifications, the equation of motion can be linearized around the orbit of the particle and the moon. Introducing the relative radial and azimuthal coordinates $x=x_{p}-x_{m}$ and $y=y_{p}-y_{m}$ (subscripts $p$ and $m$ denote particle and moon position respectively), the equations of the relative motion reduce to the Hill equations

$$
\begin{aligned}
& \ddot{x}=2 \dot{y}+3 x-3 \frac{x}{\rho^{3}} \\
& \ddot{y}=-2 \dot{x}-3 \frac{y}{\rho^{3}},
\end{aligned}
$$

where $\rho=\sqrt{x^{2}+y^{2}}$ denotes the distance between moon and particle (Hill (1878)). The time is normalized by the inverse of the Keplerian frequency $\Omega_{0}=\sqrt{G M_{c} / a_{m}^{3}}$ and the space coordinates are written in units of the Hill radius

$$
h=a_{m}\left(\frac{M_{m}}{3 M_{c}}\right)^{1 / 3},
$$

where $a_{m}, G, M_{c}$ and $M_{m}$ denote the semimajor axis of the moon, the gravitational constant, the mass of the central body and the mass of the moon, respectively. The origin of the relative coordinate system is located at the center of mass of moon and particle which we identify with the position the moon in our case $\left(x^{*}=x_{m}\right.$ and $\left.y^{*}=y_{m}\right)$. The equation of motion for the center of mass can be written in the form

$$
\begin{aligned}
& \ddot{x}^{*}=2 \dot{y}^{*}+3 x^{*} \\
& \ddot{y}^{*}=-2 \dot{x}^{*} .
\end{aligned}
$$

Relative and center of mass coordinates are favorable because we can translate the problem of moon and particle being on eccentric orbits to a problem where only the particle is on 
an eccentric orbit. (Only the relative coordinates depend on the moonlet perturbation.) An effective eccentricity and longitude of pericenter can be identified with the orbit in the relative coordinate system, as defined later (see eq. (41)-(42) in section 2.4). After solving the set of equations (1)-(2) and (4)-(5) the particle postions can be reconstructed in the form $x_{p}=x+x^{*}$ and $y_{p}=y+y^{*}$.

\subsection{Unperturbed Solution}

In the asymptotic limit the distance between moon and particle becomes infinity $(\rho \rightarrow \infty)$. This simplifies equations (1)-(2) to the same form as equations (4)-(5). In this case one can write the unperturbed solution of positions and velocities as

$$
\begin{aligned}
& x(t)=C+A \sin t+B \cos t \\
& y(t)=D-\frac{3}{2} C t+2 A \cos t-2 B \sin t \\
& u(t)=A \cos t-B \sin t \\
& v(t)=-\frac{3}{2} C-2 A \sin t-2 B \cos t
\end{aligned}
$$

with the constants of integration $A, B, C$ and $D$. The solution of the center of mass motion has the same form as (6)-(9), only that the variables and parameters are labeled by the superscript *. The solution (6)-(9) is linear in the constants of integration. Therefore, one can easily relate the constants $A, A^{*}, A_{m}$ and $A_{p}$ to each other by $A_{m}=A^{*}$ and $A_{p}=A^{*}+A$ and the same holds for the constants $B, C$ or $D$.

In this paper the scaled eccentricities $e_{i}=\tilde{e}_{i} a_{m} / h$ as well as the relations

$$
\begin{aligned}
& A_{i}=-e_{i} \sin \tau_{i} \\
& B_{i}=-e_{i} \cos \tau_{i}
\end{aligned}
$$

are used, where $\tau_{i}$ denotes the longitude of pericenter and index $i$ stands for $p$ or $m$ denoting 
particle and moon parameters, respectively. The semimajor axes $a_{i}$ and the unscaled eccentricities $\tilde{e}_{i}$ of particle and moon in physical units are related to the constants $A_{i}, B_{i}$, $C_{i}$ by the equations

$$
\begin{aligned}
a_{i} & =a_{m}+h C_{i} \\
\tilde{e}_{i} & =\frac{h}{a_{m}} \sqrt{A_{i}^{2}+B_{i}^{2}},
\end{aligned}
$$

where $C_{m}$ is set to zero.

\subsection{Asymptotic solution of a moon-particle encounter for a large impact parameter}

The solution of the perturbed equations (1)-(2) is derived by the method of the variation of the constants $A, B, C$ and $D$ as they appear in the solutions of the unperturbed solutions. Thus, this set of parameters becomes time dependent and a set of differential equations is obtained for their time evolution using the equations (6)-(9)

$$
\begin{aligned}
\dot{A} & =F_{x} \cos t-2 F_{y} \sin t \\
\dot{B} & =-F_{x} \sin t-2 F_{y} \cos t \\
\dot{C} & =2 F_{y} \\
\dot{D} & =-2 F_{x}+3 F_{y} t
\end{aligned}
$$

The radial and azimuthal forces are denoted by $F_{x}=-3 x / \rho^{3}$ and $F_{y}=-3 y / \rho^{3}$, respectively.

If prior to the encounter the moon and ring particle move on circular orbits their radial separation $x=x_{p}-x_{m}=C_{p}-C_{m}=C$ can be identified with the impact parameter. Here, the notation "impact parameter" will be used for $C$ also in the more general context of elliptical orbits. 
We aim at deriving a solution that is valid for large impact parameters such that $C \gg 1, C \gg A, C \gg B$ and $C \gg D$. Thus, the perturbations of the particle orbits are small and an approximate solution can be derived in powers of the inverse impact parameter. Although, the condition $C \gg D$ is formally not fullfilled in general, the problem can be avoided if a new time variable is introduced, shifting t by a constant,

$$
t^{\prime}=t-\frac{2 D_{0}}{3 C_{0}}
$$

and defining a new set of parameters as

$$
\begin{aligned}
& A^{\prime}=A \cos \left(\frac{2 D_{0}}{3 C_{0}}\right)-B \sin \left(\frac{2 D_{0}}{3 C_{0}}\right) \\
& B^{\prime}=A \sin \left(\frac{2 D_{0}}{3 C_{0}}\right)+B \cos \left(\frac{2 D_{0}}{3 C_{0}}\right) \\
& C^{\prime}=C \\
& D^{\prime}=D-D_{0} .
\end{aligned}
$$

The solutions (6)-(9) must be rewritten accordingly

$$
\begin{aligned}
& x\left(t^{\prime}\right)=C^{\prime}+A^{\prime} \sin t^{\prime}+B^{\prime} \cos t^{\prime} \\
& y\left(t^{\prime}\right)=-\frac{3}{2} C^{\prime} t+2 A^{\prime} \cos t^{\prime}-2 B^{\prime} \sin t^{\prime} . \\
& u\left(t^{\prime}\right)=A^{\prime} \cos t^{\prime}-B^{\prime} \sin t^{\prime} \\
& v\left(t^{\prime}\right)=D^{\prime}-\frac{3}{2} C^{\prime}-2 A^{\prime} \sin t^{\prime}-2 B^{\prime} \cos t^{\prime} .
\end{aligned}
$$

The parameters $C_{0}$ and $D_{0}$ denote the initial values of $C$ and $D$, respectively. For a sufficiently large impact parameter the changes in $D^{\prime}$ are small and are negligible compared to $C^{\prime}$. Additionally, a modified longitude of pericenter $\tau^{\prime}=\tau-2 D_{0} /\left(3 C_{0}\right)$ can be defined, whereas the eccentricity remains unchanged $e^{\prime}=e$. The equations (14)-(17) keep their form when exchanging $A, B, C, D$ and $t$ by $A^{\prime}, B^{\prime}, C^{\prime}, D^{\prime}$ and $t^{\prime}$.

Next the forces $F_{x}$ and $F_{y}$ are expanded in terms of $C_{0}^{\prime-1}$ and rewritten using the equations (23)-(26). The condition of a large impact parameter guarantees a small deflection 
of the particle. Thus, the parameters $A^{\prime}, B^{\prime}$ and $C^{\prime}$ on the right hand side of the equations (23)-(26) can be approximated by the initial values $A_{0}^{\prime}, B_{0}^{\prime}, C_{0}^{\prime}$, where $D_{0}^{\prime}$ equals zero. The lengthy equations for the expanded forces are given in Appendix A (eqs. (A1)-(A2)). The equations (14)-(17) can now be expanded in the form

$$
\begin{aligned}
\dot{A}^{\prime} & =\left\{\frac{1}{C_{0}^{\prime 2}} \cdot a_{1}\left(t^{\prime}\right)+\frac{A_{0}^{\prime}}{C_{0}^{\prime 3}} \cdot a_{2}\left(t^{\prime}\right)+\frac{B_{0}^{\prime}}{C_{0}^{\prime 3}} \cdot a_{3}\left(t^{\prime}\right)+\frac{A_{0}^{\prime 2}}{C_{0}^{\prime 4}} \cdot a_{4}\left(t^{\prime}\right)+\frac{A_{0}^{\prime} B_{0}^{\prime}}{C_{0}^{\prime 4}} \cdot a_{5}\left(t^{\prime}\right)\right. \\
& \left.+\frac{B_{0}^{\prime 2}}{C_{0}^{\prime 4}} \cdot a_{6}\left(t^{\prime}\right)\right\} \cdot \operatorname{sign}\left(C_{0}^{\prime}\right)+O\left(C_{0}^{\prime-5}\right) \\
\dot{B}^{\prime} & =\left\{\frac{1}{C_{0}^{\prime 2}} \cdot b_{1}\left(t^{\prime}\right)+\frac{A_{0}^{\prime}}{C_{0}^{\prime 3}} \cdot b_{2}\left(t^{\prime}\right)+\frac{B_{0}^{\prime}}{C_{0}^{\prime 3}} \cdot b_{3}\left(t^{\prime}\right)+\frac{A_{0}^{\prime 2}}{C_{0}^{\prime 4}} \cdot b_{4}\left(t^{\prime}\right)+\frac{A_{0}^{\prime} B_{0}^{\prime}}{C_{0}^{\prime 4}} \cdot b_{5}\left(t^{\prime}\right)\right. \\
& \left.+\frac{B_{0}^{\prime 2}}{C_{0}^{\prime 4}} \cdot b_{6}\left(t^{\prime}\right)\right\} \cdot \operatorname{sign}\left(C_{0}^{\prime}\right)+O\left(C_{0}^{\prime-5}\right) \\
\dot{C}^{\prime} & =\left\{\frac{1}{C_{0}^{\prime 2}} \cdot c_{1}\left(t^{\prime}\right)+\frac{A_{0}^{\prime}}{C_{0}^{\prime 3}} \cdot c_{2}\left(t^{\prime}\right)+\frac{B_{0}^{\prime}}{C_{0}^{\prime 3}} \cdot c_{3}\left(t^{\prime}\right)+\frac{A_{0}^{\prime 2}}{C_{0}^{\prime 4}} \cdot c_{4}\left(t^{\prime}\right)+\frac{A_{0}^{\prime} B_{0}^{\prime}}{C_{0}^{\prime 4}} \cdot c_{5}\left(t^{\prime}\right)\right. \\
& \left.+\frac{B_{0}^{\prime 2}}{C_{0}^{\prime 4}} \cdot c_{6}\left(t^{\prime}\right)\right\} \cdot \operatorname{sign}\left(C_{0}^{\prime}\right)+O\left(C_{0}^{\prime-5}\right) \\
\dot{D}^{\prime} & =\left\{\frac{1}{C_{0}^{\prime 2}} \cdot d_{1}\left(t^{\prime}\right)+\frac{A_{0}^{\prime}}{C_{0}^{\prime 3}} \cdot d_{2}\left(t^{\prime}\right)+\frac{B_{0}^{\prime}}{C_{0}^{\prime 3}} \cdot d_{3}\left(t^{\prime}\right)+\frac{A_{0}^{\prime 2}}{C_{0}^{\prime 4}} \cdot d_{4}\left(t^{\prime}\right)+\frac{A_{0}^{\prime} B_{0}^{\prime}}{C_{0}^{\prime 4}} \cdot d_{5}\left(t^{\prime}\right)\right. \\
& \left.+\frac{B_{0}^{\prime 2}}{C_{0}^{\prime 4}} \cdot d_{6}\left(t^{\prime}\right)\right\} \cdot \operatorname{sign}\left(C_{0}^{\prime}\right)+O\left(C_{0}^{\prime-5}\right)
\end{aligned}
$$

using the expanded forces, where the coefficients $a_{i}\left(t^{\prime}\right), b_{i}\left(t^{\prime}\right), c_{i}\left(t^{\prime}\right)$ and $d_{i}\left(t^{\prime}\right)$ with $i=1 \ldots 6$ are given in Appendix A (eqs. (A4)-(A27)). This expansion up to the order $C_{0}^{\prime-4}$ is an extension of the solution derived by Moons et al. (1988) and increases significantly the accuracy in the applications considered later.

One can calculate the net effect of the encounter by integrating the equations from $-\infty$ to $+\infty$, which yields a correction to the unperturbed solution in the form

$$
\begin{aligned}
\Delta A^{\prime} & =\left\{\mathcal{A}_{1} \frac{1}{C_{0}^{\prime 2}}+\mathcal{A}_{3} \frac{B_{0}^{\prime}}{C_{0}^{\prime 3}}+\mathcal{A}_{4} \frac{A_{0}^{\prime 2}}{C_{0}^{\prime 4}}+\mathcal{A}_{6} \frac{B_{0}^{\prime 2}}{C_{0}^{\prime 4}}\right\} \cdot \operatorname{sign}\left(C_{0}^{\prime}\right) \\
\Delta B^{\prime} & =\left\{\mathcal{B}_{2} \frac{A_{0}^{\prime}}{C_{0}^{\prime 3}}+\mathcal{B}_{5} \frac{A_{0}^{\prime} B_{0}^{\prime}}{C_{0}^{\prime 4}}\right\} \cdot \operatorname{sign}\left(C_{0}^{\prime}\right) \\
\Delta C^{\prime} & =\left\{\mathcal{C}_{2} \frac{A_{0}^{\prime}}{C_{0}^{\prime 3}}+\mathcal{C}_{5} \frac{A_{0}^{\prime} B_{0}^{\prime}}{C_{0}^{\prime 4}}\right\} \cdot \operatorname{sign}\left(C_{0}^{\prime}\right)
\end{aligned}
$$




$$
\Delta D^{\prime}=\left\{\mathcal{D}_{1} \frac{1}{C_{0}^{\prime 2}}+\mathcal{D}_{3} \frac{B_{0}^{\prime}}{C_{0}^{\prime 3}}+\mathcal{D}_{4} \frac{A_{0}^{\prime 2}}{C_{0}^{\prime 4}}+\mathcal{D}_{6} \frac{B_{0}^{\prime 2}}{C_{0}^{\prime 4}}\right\} \cdot \operatorname{sign}\left(C_{0}^{\prime}\right)
$$

where the constants $\mathcal{A}_{i}, \mathcal{B}_{i}, \mathcal{C}_{i}$ and $\mathcal{D}_{i}$ correspond to the integrated coefficients $a_{i}\left(t^{\prime}\right), b_{i}\left(t^{\prime}\right)$, $c_{i}\left(t^{\prime}\right)$ and $d_{i}\left(t^{\prime}\right)$. Their numerical values are listed in Table 4 in Appendix A.

In principle one can use the solutions (31) - (34) to derive the second order correction from the equations (27)-(30), but this would lead to terms of order $C^{\prime-5}$ and smaller, which are already neglected.

All integrations over $t^{\prime}$ yield finite numbers except for the term $\mathcal{D}_{1}$ which corresponds to the diverging integral

$$
\mathcal{D}_{1}=\int_{-\infty}^{+\infty} 6\left(1+9 t^{\prime 2} / 4\right)^{-1 / 2} d t^{\prime}
$$

This divergence is an artifact of neglecting the curvature in the Hill equations. However, we can choose the initial $D_{0}$ arbitrarily since we are interested only in the closer vicinity of the moon. The problem with the divergence can be avoided by integrating the considered term only from $t_{\text {min }}^{\prime}$ to $t^{\prime}$. Then $\mathcal{D}_{1}$ can be rewritten in the form

$$
\mathcal{D}_{1}\left(t^{\prime}\right)=4 \operatorname{arcsinh}\left(\frac{3}{2} t^{\prime}\right)-4 \operatorname{arcsinh}\left(\frac{3}{2} t_{\text {min }}^{\prime}\right) .
$$

Using this approach, the factor $\mathcal{D}_{1}\left(t^{\prime}\right)$ is very small for all applications in the paper and will henceforth be neglected (see Appendix B).

At this point it should be noted that the solution derived here is also applicable for encounters of satellites with arbitrary mass ratio (Henon and Petit (1986)).

\subsection{Streamline equations}

The investigations presented above serve as a base to construct the wake-streamlines, in an analogous manner as for the circular model (Showalter et al. (1986)). The streamline 
solution is restricted to orders $C^{-3}$ in the main text. However, the order $C^{-4}$ can be derived in the same way and is given in Appendix C.

The origin of our coordinate system is fixed to the gyrocenter of the moon so that $C_{m}=0$ and $D_{m}=0$. Further, the initial positions of the particles' gyrocenters are defined by $x_{0}$ and $y_{0}$, and thus $C_{p 0}=x_{0}$ and $D_{p 0}=y_{0}$. For our purpose a streamline is defined as a line which includes all possible positions of particles with a fixed parameter $x_{0}$ (at a certain time $t$ ), where $y_{0}$ varies from $-\infty$ to $+\infty$.

Initial particle eccentricities $e_{p 0}$ can be caused by resonant perturbations, for example. They are induced by a satellite exterior to the rings and characterized by a mean eccentricity and common phase for all ring particles in a small ring annulus. Thermal excitations correspond to a random component for the initial particle eccentricity and an associated uniformly distributed longitude of pericenter. Summarizing, the following expressions for the initial values of the system apply

$$
\begin{array}{ll}
A_{0}=A_{p 0}-A_{m} & =-e_{p, 0} \sin \tau_{p}+e_{m} \sin \tau_{m} \\
B_{0}=B_{p 0}-B_{m} & =-e_{p, 0} \cos \tau_{p}+e_{m} \cos \tau_{m} \\
C_{0}=C_{p 0} & =x_{0} \\
D_{0} & =D_{p 0}=y_{0} .
\end{array}
$$

In practice it is convenient to use an effective eccentricity $e$ and an effective longitude of pericenter $\tau$ which are related to $e_{p}, \tau_{p}, e_{m}$ and $\tau_{m}$ by the equations

$$
\begin{aligned}
e^{2} & =e_{p}^{2}+e_{m}^{2}-2 e_{p} e_{m} \cos \left(\tau_{p}-\tau_{m}\right) \\
\tan \tau & =\frac{-e_{p} \sin \tau_{p}+e_{m} \sin \tau_{m}}{-e_{p} \cos \tau_{p}+e_{m} \cos \tau_{m}}
\end{aligned}
$$

The initial effective eccentricity and effective longitude of pericenter are denoted by $e_{0}$ and $\tau_{0}$, respectively. 
Applying the initial conditions to the solutions (31)-(34) we write

$$
\begin{aligned}
\Delta A^{\prime} & =\left\{\frac{\mathcal{A}_{1}}{x_{0}^{2}}-\frac{\mathcal{A}_{3}}{x_{0}^{3}} e_{0} \cos \left(\tau-\frac{2 y_{0}}{3 x_{0}}\right)\right\} \cdot \operatorname{sign}\left(x_{0}\right) \\
\Delta B^{\prime} & =\left\{-\frac{\mathcal{B}_{2}}{x_{0}^{3}} e_{0} \sin \left(\tau-\frac{2 y_{0}}{3 x_{0}}\right)\right\} \cdot \operatorname{sign}\left(x_{0}\right) \\
\Delta C^{\prime} & =\left\{-\frac{\mathcal{C}_{2}}{x_{0}^{3}} e_{0} \sin \left(\tau-\frac{2 y_{0}}{3 x_{0}}\right)\right\} \cdot \operatorname{sign}\left(x_{0}\right) \\
\Delta D^{\prime} & =\left\{\frac{\mathcal{D}_{1}}{x_{0}^{2}}-\frac{\mathcal{D}_{3}}{x_{0}^{3}} e \cos \left(\tau-\frac{2 y_{0}}{3 x_{0}}\right)\right\} \cdot \operatorname{sign}\left(x_{0}\right),
\end{aligned}
$$

using the transformation $\tau^{\prime}=\tau-2 y_{0} /\left(3 x_{0}\right)$. Now the post-encounter parameter $A^{\prime}=A_{0}^{\prime}+\Delta A^{\prime}$ can be determined $\left(B^{\prime}, C^{\prime}\right.$ and $D^{\prime}$ accordingly). Using them together with the transformations (18)-(22) and the solutions (23)-(24) one can calculate the equations for the particle trajectories

$$
\begin{aligned}
x_{p}\left(x_{0}, y_{0}, t\right)= & x_{0}-e_{p, 0} \cos \left(t-\tau_{p 0}\right)+\operatorname{sign}\left(x_{0}\right)\left\{-\frac{\mathcal{C}_{2}}{x_{0}^{3}} e_{0} \sin \left(\tau-\frac{2 y_{0}}{3 x_{0}}\right)\right. \\
+ & {\left[\frac{\mathcal{A}_{1}}{x_{0}^{2}}-\frac{\mathcal{A}_{3}}{x_{0}^{3}} e_{0} \cos \left(\tau-\frac{2 y_{0}}{3 x_{0}}\right)\right] \sin \left(t-\frac{2 y_{0}}{3 x_{0}}\right) } \\
& \left.-\frac{\mathcal{B}_{2}}{x_{0}^{3}} e_{0} \sin \left(\tau-\frac{2 y_{0}}{3 x_{0}}\right) \cos \left(t-\frac{2 y_{0}}{3 x_{0}}\right)\right\} \\
y_{p}\left(x_{0}, y_{0}, t\right)= & y_{0}-\frac{3}{2} x_{0} t+2 e_{p, 0} \sin \left(t-\tau_{p 0}\right)+\left\{\frac{\mathcal{D}_{1}}{x_{0}^{2}}-\frac{\mathcal{D}_{3}}{x_{0}^{3}} e_{0} \cos \left(\tau-\frac{2 y_{0}}{3 x_{0}}\right)\right. \\
& -\left[y_{0}-\frac{3}{2} x_{0} t\right] \cdot \frac{\mathcal{C}_{2}}{x_{0}^{4}} e_{0} \sin \left(\tau-\frac{2 y_{0}}{3 x_{0}}\right)+2 \frac{\mathcal{B}_{2}}{x_{0}^{3}} e_{0} \sin \left(\tau-\frac{2 y_{0}}{3 x_{0}}\right) \sin \left(t-\frac{2 y_{0}}{3 x_{0}}\right) \\
& \left.+2\left[\frac{\mathcal{A}_{1}}{x_{0}^{2}}-\frac{\mathcal{A}_{3}}{x_{0}^{3}} e_{0} \cos \left(\tau-\frac{2 y_{0}}{3 x_{0}}\right)\right] \cos \left(t-\frac{2 y_{0}}{3 x_{0}}\right)\right\} \operatorname{sign}\left(x_{0}\right) .
\end{aligned}
$$

The equations (47) - (48) describe the particle trajectories depending on time $t$. However for fixed $t$ they also describe the streamlines, if the particles' dispersion velocity is much smaller than the moonlet induced velocity. In this case the parameter which has to be varied is $y_{0}$. Particle trajectory and streamline generally deviate from each other because the streamline changes its form in time, which is equivalent to the statement that the flow of ring material is no longer stationary. 
The solution (47) for the x-component of the motion is valid to order $C^{-3}$. It was first derived by Julian and Toomre (1966) in the context of stellar discs to the order $C^{-2}$, and Goldreich and Tremaine (1980) gave a similar solution in context of planetary rings. Moons et al. (1988) derived a solution for semimajor axis and eccentricity vector up to order $C^{-3}$.

\subsection{Comparison of analytical and numerical solution}

In this section the effect of initially eccentric orbits on the shape of the streamlines is illustrated. Further, the analytical model is compared with a full numerical integration of the equations of motion (1)-(2).

Figure 1 shows streamlines for different moon eccentricities $e_{m}$ at $x_{0}=4.5$ and $x_{0}=6.0$, respectively. The eccentricity of the particles $e_{p, 0}$ is set to zero, only $e_{m}$ and $\tau_{m}$ are varied. However, this does not affect the comparison of analytical and numerical solution which is of major interest here. The top panels of figure $1\left(e_{m}=0\right)$ show that analytical and numerical solution approach each other after a quarter orbital period. Then the particles are distant enough from the moon so that the asymptotic solution gives a fair approximation. For larger initial eccentricities streamlines deviate more from the sinusoidal shape. For small initial eccentricities $e_{m}$, however, the analytical solution provides a good approximation $\left(e_{m}<0.5\right.$ for $x_{0}=4.5$ and $e_{m}<2$ for $\left.x_{0}=6.0\right)$. Deviations become larger with increasing azimuthal distances $y$, caused by the Keplerian shear which amplifies initially small deviations of $C$, meaning that the azimuthal separation of two particles on one streamline increases downstream.

Please, insert figure 1 HeRe.

The strength of the moonlet's gravity felt by a particle varies additionally with the epicyclic motion of the moon if the moonlet moves on an eccentric orbit. Thus, the 
agreement between analytics and numerics also dependends on the longitude of pericenter $\tau_{m}$. Figure 2 shows the streamlines for different moon phases $\tau_{m}$ at $x_{0}=4.5$ and $x_{0}=6.0$ where $e_{m}=1.0$ has been chosen. In both cases the analytics shows a fair agreement with the numerical solutions, while, as expected, the quality of the analytical approximation depends on $\tau_{m}$. The postion of the moon on its epicycle only dependends on the difference $t-\tau_{m}$. Thus, plots for different $\tau_{m}$ can also be interpreted as snapshots taken at different times $t$ but constant $\tau_{m}$, and thus figure 2 also shows the shape of the streamlines at different times.

\section{Please, insert Figure 2 HeRE.}

In a next step the applicability of the analytical solution is quantified in dependence on the model parameters (impact parameter $x_{0}$, initial effective eccentricity $e_{0}$, initial effective pericenter of longitude $\left.\tau_{0}\right)$. For this purpose a measurement $s\left(x_{0}, e_{0}, \tau\right)$ is introduced by calculating the maximum deviation between analytical $x_{a}\left(y_{i}\right)$ and numerical $x_{n}\left(y_{i}\right)$ solution.

$$
s\left(x_{0}, e_{0}, \tau\right)=\left|\frac{\max \left(x_{a}\left(y_{i}\right)-x_{n}\left(y_{i}\right)\right)}{\max \left(x_{n}\left(y_{i}\right)\right)-\min \left(x_{n}\left(y_{i}\right)\right)}\right|
$$

In order to relate the deviation to a measureable quantity the deviation is normalized by the radial amplitude of the streamline. Note that the maximal deviation between analytical and numerical solution is used here rather and not the standard deviation. This results in a more conservative estimate providing higher values for $s\left(x_{0}, e_{0}, \tau\right)$.

Figure 3 shows three contour plots of $s\left(x_{0}, e_{0}, \tau\right)$ for different $x_{0}$. As expected, the deviation $s$ is generally an increasing function of the effective eccentricity $e_{0}$ and a decreasing function of $x_{0}$. Further, $s$ also depends on $\tau_{0}$ and has its largest values for $\pi / 4<\tau_{0}<\pi / 2$. As a conservative estimate, the maximum of $s$ with respect to $\tau_{0}$ is calculated. The results are plotted in figure 4 versus $e_{0}$ and $x_{0}$, where analytical solutions are used to order $x_{0}^{-3}$ (left panel) and $x_{0}^{-4}$ (right panel). From figures 1 and 2 we conclude that $e_{m}=0.5$ for 
$x_{0}=4.5$ and $e_{m}=2$ for $x_{0}=6.0$ are still fair approximations. Comparing to figure 4 (right panel) this suggests that $s>0.15$ gives a rough boundary for the applicability of the model. The condition

$$
x_{0}>x_{0, \mathrm{crit}}=0.25 \cdot e^{2}+0.8 \cdot e+3.5
$$

can then be used as a simple approximation of the isoline $s=0.15$. For the order $x_{0}^{-3}$ solution the deviations of the analytical model from the exact solution are larger $(s=0.3$ rather than $s=0.15)$; to get the same accuracy $s=0.15$ with the $x_{0}^{-3}$ solution one is restricted to $x_{0}>x_{0, \text { crit }}=0.43 \cdot e^{2}+1.4 \cdot e+3.5$. Comparison between this criterion and equation (50) allows to decide if a 3rd or 4th order solution is needed for a given set of parameters. Summarizing, the analytical model of order $x_{0}^{-4}$ is well applicable at least within $15 \%$ ( $<2 \%$ mean deviation) as long as condition (50) is fullfilled.

Please, insert figure 3 HeRe.

Please, insert Figure 4 Here.

\section{Streamline wake model}

From the analytical expression for the streamlines, we can calculate the pattern in the surface mass density of the rings in the region of the moonlet induced wakes. This is of special interest because an anlytical expression of the expected density (or optical depth) can be compared to optical depth profiles from stellar and radio occultation.

As mentioned before, the streamlines are no longer stationary for the case of an eccentric moon, and they do not coincide with the particle trajectories as it is the case for stationary streamline models of pertubed planetary rings (Borderies et al. (1982)). Nevertheless, the density can be determined in the same way as for the stationary case. 
Figure 5 shows families of streamlines at different times t. The moon parameters are set to $e_{m}=0.75$ and $\tau_{m}=0$. Due to the radial eccentricity gradient of the particles, and especially due to the Kepler shear, there are regions where the distance of neighboring streamlines varies locally, corresponding to regions of higher and lower densities. In this picture the streamlines can be interpreted as mass loaded lines. Streamlines can cross in our simple kinematic model, which would lead to a diverging density. In reality, particle interactions avoid such a behavior, bending the shape of the streamlines, so that they cannot cross.

\section{Please, insert Figure 5 HeRE.}

The fact that surface density increases, when streamlines approach locally, is mathematically described by the Jacobian determinant (or compression factor; Borderies et al. (1982))

$$
J=\left|\begin{array}{ll}
\frac{\partial\left(x_{s}\right)}{\partial\left(x_{0}\right)} & \frac{\partial\left(x_{s}\right)}{\partial\left(y_{0}\right)} \\
\frac{\partial\left(y_{s}\right)}{\partial\left(x_{0}\right)} & \frac{\partial\left(y_{s}\right)}{\partial\left(y_{0}\right)}
\end{array}\right|=\left|\frac{\partial\left(x_{s}\right)}{\partial\left(x_{0}\right)} \frac{\partial\left(y_{s}\right)}{\partial\left(y_{0}\right)}-\frac{\partial\left(y_{s}\right)}{\partial\left(x_{0}\right)} \frac{\partial\left(x_{s}\right)}{\partial\left(y_{0}\right)}\right|
$$

Then, the perturbed surface density $\Sigma$ is defined as

$$
\Sigma=J^{-1} \Sigma_{0}
$$

where $\Sigma_{0}$ denotes the unperturbed density. Using the steamline equations (47)-(48) the Jacobian can be determined, replacing $x_{0}$ and $y_{0}$ by the guiding center coordinates $X=x_{0}$ and $Y=y_{0}-3 x_{0} / 2$

$$
\begin{aligned}
J(X, Y, t)= & 1-\operatorname{sign}(X)\left\{\frac{2 \mathcal{C}_{2} e \sin \left(t-\tau+\frac{2 Y}{3 X}\right)}{X^{4}}+\frac{2 \mathcal{D}_{3} e \sin \left(t-\tau+\frac{2 Y}{3 X}\right)}{3 X^{4}}\right. \\
& +\sin \left(\frac{2 Y}{3 X}\right)\left[\frac{2 \mathcal{A}_{1}}{3 X^{3}}+\frac{\left(4 \mathcal{B}_{2}-5 \mathcal{A}_{3}\right) e \cos \left(t-\tau+\frac{2 Y}{3 X}\right)}{3 X^{4}}\right. \\
& \left.+\frac{2\left(\mathcal{A}_{3}+\mathcal{B}_{2}\right) e Y \sin \left(t-\tau+\frac{2 Y}{3 X}\right)}{3 X^{5}}\right]+\cos \left(\frac{2 Y}{3 X}\right)\left[\frac{2 \mathcal{A}_{1} Y}{3 X^{4}}\right.
\end{aligned}
$$




$$
\left.-\frac{2\left(\mathcal{A}_{3}+\mathcal{B}_{2}\right) e Y \cos \left(t-\tau+\frac{2 Y}{3 X}\right)}{3 X^{5}}+\frac{\left(4 \mathcal{A}_{3}-5 \mathcal{B}_{2}\right) e \sin \left(t-\tau+\frac{2 Y}{3 X}\right)}{3 X^{4}}\right](5.3)
$$

Guiding center coordinates are convenient to describe the density because they approximate the radial $x$ and azimuthal $y$ coordinates in zeroth order and differ from them just by the moon induced eccentricity $\approx \mathcal{A}_{1} / X^{2}$ if one neglects initial particle eccentricities. Of course, if one has systematic initial particle eccentricties, the guiding centers correspond in zeroth order to $X=x+e_{p, 0} \cos \left(t-\tau_{p, 0}\right)$ and $Y=y-2 e_{p, 0} \cos \left(t-\tau_{p, 0}\right)$. For a more accurate solution the equations (47)-(48) have to be solved for $X$ and $Y$, which becomes important if the nonlinearity parameter (see eq. (59)), is close to unity.

The resulting pattern of the surface density is shown in figure 6 at different times $t$ where bright and dark grayscale represents high and low density regions, respectively. Here the approximation $X=x$ and $Y=y$ has been used. The position of the wake crests and their maximal surface mass density varies with time. The double peak in some density maxima is a result of the streamline crossing and could be avoided by implementing particle interactions in the model.

\section{Please, insert Figure 6 HeRE.}

Figure 7 shows the radial profiles of the wakes at different times. The positions $x$ and $y$, corresponding to density and the guiding center positions, are calculated from equations (47)-(48). Besides the crests' variations in maximal densities and positions, the whole shape is time dependent. At one time the crests appear flattend, then stretched or the profiles develop a saw tooth shape tilted once to the right and once to the left. However, the distance between the crests remains almost constant. For this figure, a zero initial ring eccentricity $e_{p}=0$ is assumed. A non-zero ring eccentricity would mainly lead to a shift of the whole density structure in radial and azimuthal direction.

Equation (53) for the compression factor $J$ can be rewritten in form of a Fourier series, 
with the eigenfunctions $\cos (n \cdot 2 Y /(3 X))$ and $\sin (n \cdot 2 Y /(3 X))$. This makes clear why the corresponding radial and azimuthal wavelengths have the form $\lambda_{x}=3 \pi X^{2} / Y \approx 3 \pi x^{2} / y$ and $\lambda_{y}=3 \pi X \approx 3 \pi x$ and are nearly independent of the moonlet eccentricity.

Please, insert figure 7 Here.

Moreover, equation (53) can also be written in the form

$$
J=1+p+q \cos \left(\frac{2 Y}{3 X}+\gamma\right)
$$

which is similar to the form used in the literature (see Borderies et al. (1982); Showalter et al. (1986)). The nonlinearity parameter and the density phase shift are denoted by $q$ and $\gamma$, respectively. Additionally, the parameter $p$ is introduced describing the density response to the change in streamline parameters $C$ and $D$. At the first non-trivial order, $X^{-3}$ (circular orbits), the Showalter et al. (1986) density solution can be reproduced, with

$$
\begin{aligned}
p & =0 \\
q & =\frac{2 \mathcal{A}_{1} \operatorname{sign}(X) Y}{3 X^{4}} \sqrt{1+\frac{X}{Y}} \\
\gamma & =\arctan \left(\frac{X}{Y}\right)
\end{aligned}
$$

In the cases of interest the azimuthal coordinate is usually much larger than the radial one $Y \gg X$ and the equations above can be simplified $\left(q=q_{0}=\left(2 \mathcal{A}_{1} \operatorname{sign}(X) Y\right) /\left(3 X^{4}\right)\right.$ and $\left.\gamma=\gamma_{0}=0\right)$. The next order solution $\left(O\left(X^{-4}\right)\right.$ and using again $\left.Y \gg X\right)$ can be written as

$$
\begin{aligned}
p & =-\frac{2\left(3 \mathcal{C}_{2}-\mathcal{D}_{3}\right)}{3 X^{4}} e \operatorname{sign}(X) \sin \left(t-\tau+\frac{2 Y}{3 X}\right) \\
q & =q_{0}\left[1-\frac{\left(\mathcal{A}_{3}+\mathcal{B}_{2}\right)}{\mathcal{A}_{1} X} e \cos \left(t-\tau+\frac{2 Y}{3 X}\right)\right] \\
\gamma & =\gamma_{0}+\frac{\left(\mathcal{A}_{3}+\mathcal{B}_{2}\right)}{\mathcal{A}_{1} X} e \sin \left(t-\tau+\frac{2 Y}{3 X}\right) .
\end{aligned}
$$

The effects of an initial eccentricity of the streamline as well as an eccentricity of the perturbing moon, are described by the parameters $p, q$ and $\gamma$, which are periodic functions 
of time. The variations of $p$ and $q$ cause variations of the wake crest maxima, where the $q$ term dominates the $p$ term in the tight winding limit $(Y \gg X)$. The phase shift $\gamma$ leads to a periodic modulation of locations of the wake maxima. However, it has no term linear in $Y$ which would correspond to a perturbation of the wavelength of the density pattern. The next order solution of $p, q$ and $\gamma$ is given in Appendix C.

The point of steamline crossing can be calculated for initially circular orbits as $X_{0, \text { crit }}=\left(2 \mathcal{A}_{1} \operatorname{sign}(X) Y / 3\right)^{1 / 4}$ from equation (56) for $Y \gg X$. The inclusion of initially eccentric orbits requires a correction to this equation, and one can use equation (59) to derive a correction at linear order of the initial eccentricity $e_{0}$. Here a conservative estimate is derived, where the cosine is set to unity which results in the equation

$$
X_{\text {crit }}=X_{0, \text { crit }}-\frac{\mathcal{A}_{3}+\mathcal{B}_{2}}{4 \mathcal{A}_{1}} \cdot \frac{e_{0}}{X_{0, \text { crit }}} \operatorname{sign}\left(X_{0, \text { crit }}\right) .
$$

\section{Streamwire model}

The model presented in the previous section is well suitable to describe the perturbed ring density beyond the gap edges, but not directly at the edges where the initial density drops off. Addionally, the streamline model neglects the thermal velocity of the particles compared to the systematic moonlet induced velocity. In order to lift these restrictions, Stewart (1991) introduced a nonlinear wake model considering the effect of velocity dispersion. He derived a particle phase space distribution and calculated the zero and first moments in terms of a series expansion. The thermal motion leads to a displacement of the particles from the streamline giving the streamline a finite width. In this case the streamlines become steamwires and as a consequence the density keeps finite when the streamwires cross or merge. In this section the concept of streamwires is used to calculate the surface density at the gap edge. 


\subsection{Boltzmann equation and characteristic equations}

The phase space distribution of the particles $f(\vec{r}, \vec{v}, t)$ (describing for example the velocity dispersion) and its evolution is determined by the Boltzmann equation

$$
\partial_{t} f+\vec{v} \cdot \nabla_{\vec{r}} f+\vec{G} \cdot \nabla_{\vec{v}} f=\left(\partial_{t} f\right)_{\text {coll }}
$$

where

$$
\vec{G}=\left(\begin{array}{c}
2 v+3 x+F_{x} \\
-2 u+F_{y}
\end{array}\right)
$$

denotes the generalized specific force field (force per mass) as used in equation (1) and (2). The change of the distribution due to particle collisions is represented by $\left(\partial_{t} f\right)_{\text {coll }}$.

The Boltzmann equation is an integro-differential equation. However, collisions are neglected in the following consideration. Thus, the equation reduces to a first order partial differential equation, which can be solved with the method of characteristics. The partial differential equation can be written in terms of the total derivative of $f(\vec{r}, \vec{v}, t)$

$$
\frac{d f}{d \tilde{t}}=\frac{d t}{d \tilde{t}} \partial_{t} f+\frac{d \vec{r}}{d \tilde{t}} \cdot \nabla_{\vec{r}} f+\frac{d \vec{v}}{d \tilde{t}} \cdot \nabla_{\vec{v}} f
$$

Comparing the equations (62) and (64) the characteristic equations can be obtained

$$
\begin{aligned}
& \frac{d f}{d \tilde{t}}=\left\langle\partial_{t} f\right\rangle_{\text {coll }}=0 \\
& \frac{d t}{d \tilde{t}}=1 \\
& \frac{d \vec{r}}{d \tilde{t}}=\vec{v} \\
& \frac{d \vec{v}}{d \tilde{t}}=\vec{G}
\end{aligned}
$$

where $\tilde{t}$ is the parameter of the characteristics. Because we neglect collisions, $d f / d \tilde{t}$ becomes zero and the solution can be derived from the initial condition $f=f_{0}$. The solution of the second equation (66) corresponds to equation (18) which means $\tilde{t}=t^{\prime}$. The last two characteristic equations are the Hill equations, as constructed, and the solutions for the spatial directions are given by equations (47)-(48). 


\subsection{Phase-space distribution of the particles}

The initial unperturbed phase space distribution is assumed to be a vertically integrated Rayleigh distribution

$$
f_{0}\left(e_{\mathrm{th}}, \tau_{\mathrm{th}}, X, Y\right)=\frac{2 e_{\mathrm{th}}}{\left\langle e^{2}\right\rangle} \exp \left\{-\frac{e_{\mathrm{th}}^{2}}{\left\langle e^{2}\right\rangle}\right\}
$$

where $e_{\mathrm{th}}$ denotes the eccentricity induced by the thermal motion of particles and the initial phase $\tau_{\text {th }}$ is uniformly distributed. The distribution $f_{0}$ is normalized to unity and the standard deviation $\left\langle e^{2}\right\rangle$ of $e_{\mathrm{th}}$ can be identified with the radial thermal velocity by $\left\langle e^{2}\right\rangle=2 c_{x}^{2}$ where the parameters are written in dimensionless units.

Equations (47)-(48) describe the particle positions inside the streamwire and can be written in the form

$$
\begin{aligned}
& x=x_{s}\left(X, Y, e_{0}, \tau_{0}\right)-e_{\mathrm{th}} \cos \left(t-\tau_{\mathrm{th}}\right)+O\left(e_{\mathrm{th}} / X^{-3}\right) \\
& y=y_{s}\left(X, Y, e_{0}, \tau_{0}\right)+2 e_{\mathrm{th}} \sin \left(t-\tau_{\mathrm{th}}\right)+O\left(e_{\mathrm{th}} / X^{-3}\right)
\end{aligned}
$$

The first parts $x_{s}$ and $y_{s}$ are the solution of a streamline (mean position of the streamwire) which only depend on the guiding center position and the mean initial eccentricity $e_{0}$ and longitude $\tau_{0}$. The second term represents a particle's deviation from the mean position due to the thermal motion. If the thermal velocity is much smaller than the moonlet induced velocities $\left(e_{\mathrm{th}} \ll e_{0}\right)$ equations (70)-(71) give a fair approximation. For propeller generating moonlets this does not hold in general. However, here we focus on larger ring-moons able to clear a gap.

The thermally excited eccentricity $e_{\text {th }}$ can now be gained from equations (70)-(71) and the solution can be substituted in the initial phase space distribution (69) leading to the final distribution

$$
f(x, y, X, Y)=\frac{1}{4 \pi c_{x}^{2}} \exp \left\{-\frac{1}{2 c_{x}^{2}}\left(\left[x-x_{s}(X, Y)\right]^{2}+\frac{\left[y-y_{s}(X, Y)\right]^{2}}{4}\right)\right\} .
$$




\subsection{Density at the gap edge}

The surface number density can be determined by integrating the distribution function (72) over the guiding center positions $X$ and $Y$, which has been done for the circular case by Stewart (1991). Here we are interested in the density at the gap edge, including the effect of a moon and a gap edge with a systematic initial eccentricity. In this case the equations become more complex. The integration can be done numerically by summing over all contributing $X$ and $Y$.

Figure 8 shows the resulting densities for the wakes. The mean radial position of the gap edge is set to $X=4$ with the same parameters as in figure 5 , which presents the corresponding streamlines for the calculated density. The brightness and position variations of the wake crests are already discussed in the context of figure 6 , but now addionally the density variations at the gap edges can be plotted. It should be noted that particle interactions are still neglected in this model which could alter the resulting density structure significantly downstream the moon.

Please, insert figure 8 Here.

\section{Comparison to Observations}

The streamline solutions (47)-(48) themselves can be used as a model of a gap edge or of a ringlet perturbed by a moonlet. Fitting the model to a gap edge, it would be possible to constrain the mean distance $x_{0}$ between moon and edge, the effective eccentricity $e$, the effective longitude of pericenter $\tau$ and the Hill radius $h$. Moreover, conclusions on other parameters, such as moon and edge eccentricity or mass of the moon, can also be made. Thus, modeling the gap edges provides an alternative method to determine the orbital elements and mass of the gap moon as well as the eccentricity and mean radial locations of 
the gap edges.

Studies of the Keeler gap edge have been performed by the Cassini imaging team (Tiscareno et al. (2005); Weiss et al. $(2005,2009)$ ) and results for the Hill radius of Daphnis (or corresponding moonlet mass) have been published (Porco et al. (2007)). Here, we analyze images of the Keeler gap applying a streamline model to the gap edges. In this way we determine the parameters $\left(h, x_{0}, e, \tau\right)$ which can be used (a) for a comparison with literature and (b) for future modeling. The analytical streamline solution provides a fair approximation, as long as the particles remain distant enough from the moon during the encounter, and their dispersion is small compared to the induced systematic deflection. We decided to use numerical solutions of the streamlines by integrating the Hill equations (1)-(2) in order to fit the data. Moreover, the exact numerical solution provides a check for the quality of the analytic approximation later.

The perturbed ringlets in the Encke gap, radially offset from the orbit of Pan (Burns et al. (2005)), can also be fitted by a streamline. Thus, they offer an additional constraint on the mass of Pan. The ringlets are well suited for this approach, because they consist mainly of dust with low optical depth. Thus, perturbations of the particle trajectories due to collisions can be safely neglected in contrast to the Encke gap edges. The eccentricities and longitudes of pericenter of the ringlets near the Pan orbit can also be determined for different times offering a method to test for a possible heleotropicity of these ringlets, as it has been observed for the Pan ringlet for example (Hedman et al. (2007)). However, such measurements from the ringlets are not published so far. Here, five images are investigated in order to demonstrate the applicability of the model. 


\subsection{Keeler gap edge}

We model the observed Keeler gap edge, using a streamline to represent a density isoline at the edge. We choose high resolution images of the moon Daphnis and the surrounding Keeler gap using the Planetary Data System (PDS, http://pds.jpl.nasa.gov). The images are calibrated with CISSCAL software package (version number 3.3) provided by the Imaging Science Subsystem (ISS) team (Porco et al. (2004)). Further the geometry of each image is solved, i.e. we determine the radial and azimuthal position of each image pixel in the ring using the NAIF SPICE toolkit. The list of images we investigate is given in Appendix D, Table 5, together with image time, resolution and parameters of observation geometry. The images are taken from a time span of less than two years with exposure times between 120 and $820 \mu \mathrm{s}$. The radial and azimuthal resolution varies between 2 to 7 and 4 to $14 \mathrm{~km}$ per pixel, respectively. All images are from the sunlit side of the ring, taken mostly at low phase angles $\left(<4^{\circ}\right)$. Only one ring image, we use, has a larger phase angle of $114^{\circ}$. Incidence and emission angles are comparable for all images.

The moon position is determined from the image by fitting a 2-dimensional Gaussian brightness model as well as by calculating the center of the brightness distribution. Both methods give similar results. Since, most images are taken at low phase angle a correction between calculated brightness center and real moon position is not necessary. Image N1536484175 has a phase angle of $114^{\circ}$, and thus less than one half of the sun illuminated part of the moon is visible. We use an effective radius of Daphnis, because of the nonspherical shape of the moon, in order to correct for the real position of the moon with respect to the measured brightness center. Since the ring is a good reflector of sun light, the night side of the moon is weakly illuminated and thus visible. This allows to addionally estimate the center of the moon by eye. Both correction methods give similar results for the position of Daphnis and delivered the same values for the fit of the model 
parameters. The moon positions are used as the origin of the coordinate system, so that the model fit is calculated in the center of mass system.

The brightness profile across the gap edge has been fitted with an error function model

$$
f_{\text {edge }}(i)=f_{0}+f_{1} \operatorname{erf}\left(\frac{i-i_{\text {edge }}}{\delta i}\right)
$$

(e.g. to account for a possible smear of the gap edges in the images) where the mean pixel position of the edge $i_{\text {edge }}$ along an image column and/or image row are fitted (depending on the orientation of the gap along the image) in order to determine the radial edge mean position. The parameter $\delta i$ labels the finite width of the edge as it appears in the image. The positions of the inner and outer edge are determined separately using a Levenberg-Marquardt fit. The radial and azimuthal edge positions are calculated from the fitted pixel positions and the geometry solution. Two example fits are shown in figure 9 . The fitting routine finds the gap edge without any difficulties.

Typically, the edges change their periodic appearance rapidly downstream from the moon, possibly indicating the strong influence of particle interactions on the shape of the edges. For this reason only the first wake period is used to determine the kinematic model parameters. The second period is addionally taken into account, only if the effective eccentricity is small and the impact parameter is large enough, as it is the case for example in the upper panel of figure 9.

\section{Please, insert figure 9 Here.}

Besides the moonlet other perturbations may induce structure in the gap edges with wavelengths much larger than the wake wavelength $\lambda_{\text {wake }}$. In this case, the initial particle longitude of pericenter $\tau_{p}$ at the edge can be assumed constant along the fitted part of the edge. For example the wavelength for the observed Prometheus 32:31 inner Lindblad resonance at the inner edge (Tiscareno et al. (2005)) is much larger than the scale of the 
wakes $\lambda_{\text {res }} / \lambda_{\text {wake }} \approx 130$.

Usually, one uses a gradient method to minimize the $\chi^{2}$ function in order to find the best set of model parameters (e.g. Levenberg-Marquardt fit). However our model produces many minima of the $\chi^{2}$ function making it impossible to use this approach. Thus a systematic scan has been performed over all parameters and the variance between model and data

$$
\operatorname{Var}\left(h, x_{0}, e, \tau\right)=\frac{1}{N} \sum_{n=0}^{N}\left(x_{n}-x_{\text {model }, n}\right)^{2}
$$

has been minimized (similar to the $\chi^{2}$ function).

The inner and outer edges are fitted separately and the resulting parameters and their errors are listed in the tables 1 and 2, respectively. A number of different effects can cause deviations of the model from the measured data despite the uncertainty of determing the gap edge position, as for example:

1. The streamline does not correspond exactly to a density or brightness isoline as assumed in our image fit.

2. The geometry solution for the images is not exact, which can produce systematic trends.

3. The point spread function of the camera might change the appearance of the gap edge.

The actual error is a combination of these effects. Therefore, the minimal variance, Var $_{\text {bestfit }}$, corresponding to the best fit is used as a reference. Then, we calculate the variance, $\operatorname{Var}_{\text {model }}$, between the model, using different parameters, and the best fit streamline (similar to eq. (74)). We compare this variance, $\operatorname{Var}_{\text {model }}$, with the variance of the best fit, $\operatorname{Var}_{\text {bestfit }}$, and consider all models (with a given set of parameters), which verifying the relation 
$\operatorname{Var}_{\text {model }}<\operatorname{Var}_{\text {bestfit }}$, as a suitable realization. This defines possible sets of parameters inside a interval of uncertainty (one $\sigma$ error), and thus, the uncertainties of the model parameters as listed in table 1 and 2 .

Please, insert table 1 Here.

Please, insert table 2 Here.

An average Hill radius of 5.2 and $5.0 \mathrm{~km}$ is obtained from the fits to the inner and outer edge, respectively, and we conclude a Hill radius of $5.1 \pm 0.2 \mathrm{~km}$ for the moon Daphnis. This Hill radius corresponds to a Daphnis mass of $(9.9 \pm 0.6) \cdot 10^{13} \mathrm{~kg}$ and is consistent with the value of $4.9 \mathrm{~km}$ determined by the imaging team with a similar method (Porco et al. (2007)). The average pre-encounter impact parameters, determined from the inner and outer edge are $x_{0, i}=-4.1 \pm 0.2$ and $x_{0, o}=3.2 \pm 0.1$, corresponding to 21 and $16 \mathrm{~km}$, respectively. The gravity of the moon tends effectively to push away the ring particles radially while the particle interactions counteract this process leading to a balance after one synodic period (Henon (1981); Lissauer et al. (1981); Petit and Henon (1988); Spahn and Wiebicke (1989); Tiscareno et al. (2005); Lewis and Stewart (2006)). The related mean post-encounter impact parameters are $x_{0, i}=-4.1 \pm 0.2$ and $x_{0, o}=3.2 \pm 0.1$, corresponding to 21 and $17 \mathrm{~km}$, respectively. While the mean position of the inner edge changes only slightly ( $\approx 250$ meter) the outer edge is pushed away by about $1 \mathrm{~km}$. Thus, we conclude a mean gap width of $37 \pm 2 \mathrm{~km}$ close to the moon compared to directly measured $39.5 \mathrm{~km}$ (Tiscareno et al. (2005)). Our results imply that the moon is shifted radially outward from the center of the gap, using the edge mean positions as reference. It is highly unlikely (probability of $1 / 2^{5}=0.03$ ) that Daphnis' inclination or eccentricity are responsible for the observed shift because the result is found in all fits to images taken at 5 different moments in times.

One explanation of this asymmetry may be the angular momentum transfer between 
moonlet and ring (Goldreich and Tremaine (1980); Spahn et al. (1992a)). The induced eccentricity due to the Prometheus 32:31 resonance at the inner edge could be responsible for pushing the moon outwards. The majority of the ring material orbits inside Daphnis, in principle also pushing the moon closer to the outer edge. But this effect should be negligible because of the still large distance to the outer A ring edge $(200 \mathrm{~km})$ and the drastic decrease of the efficiency of angular momentum transfer with distance from the moon.

We also determined the effective eccentricties. Based on formula (41) the moonlet and edge eccentricity can be estimated from the minimal and maximal effective eccentricity as

$$
\begin{aligned}
e_{p} & =\frac{e_{\max } \pm e_{\min }}{2} \\
e_{m} & =\frac{e_{\max } \mp e_{\min }}{2}
\end{aligned}
$$

where the upper sign is used for $e_{p}>e_{m}$ and the lower sign otherwise. Assuming our data set is sufficiently large, and that we indeed measured the maximal and minimal eccentricities we can assign to the inner edge a (scaled) eccentricity of at least $e_{p}=0.8 \pm 0.1$ and to the moon an eccentricity of $e_{m}=0.5 \pm 0.1$. This leads to a variation of the inner edge of $2 e_{p} h=8 \pm 1 \mathrm{~km}$ compared to $15 \mathrm{~km}$ determined by Tiscareno et al. (2005). The fits of the outer edge give independently $e_{p}=e_{m}=0.3 \pm 0.2$. This suggests that a variation of the outer edge might also be present, but with a large uncertainty, meaning that further measurements are needed to verify this interpretation. Such a variation has not been reported so far, but the proposed variation of the outer edge is a factor of 5 smaller than the one of the inner edge (3 km outer edge and $15 \mathrm{~km}$ inner edge), and thus, much harder to detect.

From both edges we conclude a moon eccentricity of $0.4 \pm 0.2$, resulting in an unscaled eccentricity of $\tilde{e}=(1.5 \pm 0.8) \cdot 10^{-5}$. Jacobson et al. (2008) have reported an eccentricity of $3.3 \cdot 10^{-5}$ by direct orbit observation of Daphnis. Both values agree within a factor of 2 . Better values for the eccentricities of moonlet and edges could be derived from a larger set 
of images improving the statistics.

The analyzed images have been taken over a large time span. It is difficult to predict the exact rotation periods of the particles because of the oblateness of Saturn and inter-particle interactions. Thus, we have not performed a self-consistent analysis of effective eccentricities and longitude of pericenter, and their changes in time. Finally, we conclude that the resulting small $\left|x_{0}\right|$ and large $e_{0}$ made it necessary to use a numerical solution for the fits because our analytical model is not accurate enough and can only be used to reproduce the qualitative behavior of the Keeler gap edge.

Figure 10 shows a comparison between data (crosses) and fit (solid line) for two different moments in time. Addionally, the numerical streamline solution for initially circular orbits (dotted lines) and the analytical 4th order solution (dashed line, eqns. (47)-(48) and (C1)-(C2)) are plotted. The results for the outer and inner edge are presented in the right and left column, respectively. While the numerical streamline solution does not match the data perfectly, it fits significantly better than the numerical solution for initially circular orbits (dotted lines). The analytical solution fails in case of the outer edge, because of the small impact parameter. However, it describes the behavior of the inner gap edge at least qualitatively, while it is impossible to fit a solution for initially circular orbits, because of the too large eccentricity of the inner edge.

Please, insert figure 10 Here.

Summarzing, we determined parameters for Daphnis and the Keeler gap fitting a numerical streamline model to the gap edges. Our analytical model would fail, especially in case of the outer edge, because the edges are too close to the moon mean position and the eccentricities are too large. Nevertheless, the density model derived in section 3 can be applied to the Daphnis-Keeler-gap system to analyze the wakes at larger distances from the moon. This might be partly useful to interpret occultation scans of this region. 
Measured values of moonlet mass, eccentricities and mean gap width differ moderately from those in literature. Possible reasons for that are listed below:

1. There is no one-to-one correspondence of a streamline and the gap edge.

2. The available images are taken at only five moments in times. Therefore, it is likely that the instances of maximal and minimal eccentricities have not been captured and the concluded moon and edge eccentricities represent in fact lower limits of the two values. Nevertheless, our eccentricities differ only by a factor of two in comparison to other measurements.

3. Particle self-gravity and collisions have been neglected. However, restricting the fitting method to the region before streamline crossing starts (as done here), the influence of particle interactions on the resulting pattern is small, as shown for example by Lewis and Stewart (2000).

4. The inclination of the moonlet orbit has not been considered. The vertical motion of the moon can have significant influence on the resulting structures. The vertical displacement of the ring particles can even produce visible shadows on the ring (Weiss et al. (2009)). Thus, it will be important to extend the wake model for moonlets to inclined orbits. Nevertheless, if the viewing direction of the camera is nearly perpendicular to the ring plane the influence of the moonlet inclination to the observed wake structure is less important.

\subsection{Inner Pan ringlet}

Five images are chosen to apply our model to the dusty ringlet in the Encke gap inside Pan's orbit. Important image parameters are listed again in Appendix D, Table 6. The 
images are processed with the same methods as described in section 5.1. All images have a good radial resolution $(<3 \mathrm{~km})$ and the geometry solution leads to a rectification with a small residual curvature. The first image has a large phase angle and we determine the center of Pan by cursor and eye, which is the best method because of the nonspherical shape of the moon. The other images were taken in a sequence with different exposure times. They have a very small phase angle of less than 4 degrees, and thus, the moon center can be obtained from the brightness center.

The ringlet's mean position is fitted by a Gaussian model with a linear trend

$$
f_{\mathrm{r}}(i)=f_{0}+f_{1} \cdot i+f_{2} \exp \left[-\frac{1}{2}\left(\frac{i-i_{\mathrm{r}}}{\delta i}\right)^{2}\right] .
$$

Two examples of the fits are shown in figure 11. In contrast to the first image, where the ringlet postion could be determined with small deviations, the other four figures contain large fluctuations and the resulting ringlet positions are very noisy limiting the accuracy of the fit parameters.

\section{Please, insert Figure 11 HeRe.}

The measured ringlet position is fitted to the model and the resulting parameters are summarized in Table 3 . The averraged Hill radius of $17.5 \pm 0.6 \mathrm{~km}$, we find, is slightly smaller than the value of $19.1 \pm 1.0 \mathrm{~km}$ obtained from analysis of the inner Encke gap edge (Porco et al. (2005)). Differences may in part be attributed to the effect of particle interactions affecting the gap edges. On the other hand particle interactions are negligible for the dust ringlet, that we analyze. Yet undetected moons in the Encke gap might further perturb the gap edges. The Hill radius of $17.5 \pm 0.6 \mathrm{~km}$, we find, is in good agreement with Pan's semi-axis in radial direction of $17.4 \mathrm{~km}$ (Porco et al. (2007)), determined from the moonlet shape by an ellipsoid model. This supports the idea that Pan filled its Hill sphere (Roche lobe) with accreted material (Porco et al. (2007)). 
The mean ringlet position, we find, is $-5.7 \pm 0.2$ (in Hill radii), which corresponds to a distance of $100 \pm 4 \mathrm{~km}$ from Pan's orbit, consistent with the $98 \mathrm{~km}$ given by Porco et al. (2005). The analyzed images effectively represent only two different phases of the ringlet pericenter. However, the interpretion of the maximal measured eccentricity $e=0.49 \pm 0.05$, in comparison to the Pan eccentricity of 0.11 measured by Jacobson et al. (2008) (unscaled $1.4 \cdot 10^{-5}$ ), suggest a minimal ring eccentricity of $0.38 \pm 0.05$ (unscaled $5 \cdot 10^{-5}$ ). This value corresponds to an amplitude of almost $7 \mathrm{~km}$, which is sufficiently large to use the method here applied to clarify from future images if the inner Encke gap ringlet is heliotropic or not (Hedman et al. (2007)). Addionally, the impact parameter is large and the eccentricity small enough, making an application of our analytical model possible.

Please, insert table 3 Here.

\section{Summary and Discussion}

In this paper the perturbation of a particle orbit due an encounter with a moon has been calculated, numerically and analytically, for large impact parameters. The focus lies on the effect of eccentric orbits of moon and particle prior to the encounter. The analytical solution is expressed as an expansion in terms of the inverse impact parameter $C$ to order $C^{-4}$. Our analytical results have been checked against numerical integrations of particle trajectories and their corresponding streamlines. An estimate of the applicability of the analytical model is derived in terms of the effective eccentricity and the impact parameter. The analytical solution can be used to model perturbations of gap edges and narrow ringlets by the gravity of nearby eccentric moonlets, in particular if the edge is already perturbed by other bodies.

Based on the steamline solutions the density of the perturbed ring is calculated. The 
inclusion of moonlet and particle orbits which are eccentric prior the encounter leads to variations of the density pattern. Especially, wake positions and maximal densities oscillate in time, which can be modeled by a generalized definition of the non-linearity parameter $q$ and the phase shift $\gamma$. The inclusion of eccentric orbits does not lead to a change of the wavelength between the crests. A streamwire model is used to plot the density at gap edges, allowing the inclusion of a finite velocity dispersion in the model (Stewart (1991)).

Initially eccentric orbits of moons, ringlets and gap edges are obtained by fitting a numerical streamline model. First, we applied the model to the Keeler gap edges. The numerical solution is suitable to derive the Hill radius of Daphnis, the mean radial edge positions and the eccentricities of moon and edges. Results are consistent with values reported in the literature. Our analysis implies a possible non-zero value also for the eccentricity of the outer Keeler gap edge, but because of the large uncertainties this has to be proven by further observations. Further images could improve the whole analysis in future. Due to large eccentricities and small distance of moon and gap edge the analytical model developed in this paper can only account for the qualitative behavior, but not to draw quantitative conclusions. Nevertheless, the analytical density model derived in section 3 can be applied to the Keeler gap edges, as long as the radial distance to the moon is large enough ( $>4 \mathrm{~h}$ for outer edge and $>6.5 \mathrm{~h}$ for inner edge, see eq. (50)). Thus, the analytical density model is suitable for fitting optical depth profiles gained from Cassini stellar and radio occultation experiments (UVIS, RSS, VIMS).

Application of our model to a dusty ringlet in the Encke gap, perturbed by the moon Pan, yields a smaller mass for Pan than the reported in previous studies (Porco et al. (2007)). The difference may be attributed in part to the neglect of particle interactions in the model of the edge waves of the Encke gap, as it was used to infer Pan's mass (Porco et al. (2005)). An unscaled eccentricity of at least $5 \pm 1 \cdot 10^{-5}$ is proposed for the inner 
Encke gap ringlet.

The analytical model, proposed here, is partially applicable for the F-ring. This depends on the orientation of the F-ring ellipse relative to the elliptic orbit of Prometheus, which precess at different angular velocities. This leads to strong long-term variations of the effective eccentricity. Prometheus can even penetrate the ring, making an approach that uses a series expansion for large impact parameter impossible. The parameters of the F-ring core $\left(a_{\mathrm{F}-\text { ring }}=140224 \mathrm{~km}, \tilde{e}_{\mathrm{F}-\text { ring }}=0.0025\right.$, Bosh et al. (2002) $)$ and the Prometheus $\left(a_{\text {Pro }}=139380 \mathrm{~km}, \tilde{e}_{\text {Pro }}=0.0022, m_{\text {Pro }}=1.6 \cdot 10^{17} \mathrm{~kg}\right.$, Jacobson et al. $\left.(2008)\right)$ suggest a periodic variation of the scaled effective eccentricity between 0.7 and 10. Our analytical model can be used as long as the scaled effective eccentricity is below 5 , which can be estimated by equation (50) using the mean distance between ring core and moon. This means for differential precession of the F ring and Prometheus half of the period our analytical model is applicable.

The analytical wake model including the effect of moonlet and particle eccentricities, prior to the encounter, bears several advantages. First, one can model perturbations induced by several moons independently from each other, for example in regions where moonlet wakes and resonant density waves appear together or where two moons (in a gap) induce interfering wakes. In principle this accounts also for wakes repeatedly perturbed by the same moon, as it is observed for Pan (Horn et al. (1996)). However, the approach of Spahn et al. (1994) to model multiple encounters by superposing different wake generations is sufficient since terms of the smallest non-trivial order $C^{-2}$ dominate the lower order terms we calculated here. The analytical model has a few benefits in comparison to a numerical integration, because it is faster to calculate and one can study the influences of different parameters separately in detail, as for example the changes of semimajor axis, eccentricity or longitude of pericenter. 
The analytical approach could be extended in several ways. For example, the inclusion of inclined orbits or accounting for the differences of orbital, epicyclic and vertical frequencies in the model could lead to new structures. Most interesting would probably be the inclusion of a moonlet inclination. Recent observations of the Keeler gap edge revealed that the inclination of Daphnis (Jacobson et al. (2008)) has significant influence on the shape of the perturbed edges (Weiss et al. (2009)). Also the inclusion of particle interactions (collisions or self gravity) would be of interest. This would consistently avoid the streamline crossing, and thus restrict the nonlinearity parameter $q$ to values smaller than unity. This restriction implies for the order $C^{-2}$ solution a limit of the eccentricity $e<\left|3 X^{2} /(2 Y)\right|$. The consideration of particle interactions due to inelastic collisions and self-gravity is also of importance for propeller structures induced by hundred meter sized moonlets (Spahn and Sremčević (2000); Sremčević et al. (2002); Seiß et al. (2005)). 
We thank the referees for their constructive reviews. We acknowledge the efforts of the Cassini ISS team in the design and operation of the ISS instrument. This work was supported by Deutsche Forschungsgemeinschaft (Sp 384/17-1,3) and Deutsches Zentrum für Luft und Raumfahrt (50 OH 0003).

\section{A. Expansion of the moonlet gravity forces for large impact parameter}

The components of the moonlet gravity force are $F_{x}=-3 x / \rho^{3}$ for the radial and $F_{y}=-3 y / \rho^{3}$ for the azimuthal direction, where $\rho=\sqrt{x^{2}+y^{2}}$. Equations (23)-(26) are applied to rewrite the forces in terms of the parameters $A_{0}^{\prime}, B_{0}^{\prime}, C_{0}^{\prime}$ and $D_{0}^{\prime}$ of the unperturbed solution, which is needed to calculate their time evolution. For the case of a large impact parameter, the forces can be expanded in powers of $C_{0}^{\prime-1}$ as

$$
\begin{aligned}
F_{x}= & -\frac{1}{C_{0}^{\prime 2}} \cdot 3 \xi\left(t^{\prime}\right)^{3} \\
+ & \frac{A_{0}^{\prime}}{C_{0}^{\prime 3}}\left(-27 t^{\prime} \cos \left(t^{\prime}\right) \xi\left(t^{\prime}\right)^{5}+9 \sin \left(t^{\prime}\right) \xi\left(t^{\prime}\right)^{5}-3 \sin \left(t^{\prime}\right) \xi\left(t^{\prime}\right)^{3}\right) \\
+ & \frac{B_{0}^{\prime}}{C_{0}^{\prime 3}}\left(9 \cos \left(t^{\prime}\right) \xi\left(t^{\prime}\right)^{5}-3 \cos \left(t^{\prime}\right) \xi\left(t^{\prime}\right)^{3}+27 t^{\prime} \sin \left(t^{\prime}\right) \xi\left(t^{\prime}\right)^{5}\right) \\
+ & \frac{A_{0}^{\prime 2}}{C_{0}^{\prime 4}}\left(-\frac{405}{2} t^{\prime 2} \cos \left(t^{\prime}\right)^{2} \xi\left(t^{\prime}\right)^{7}+18 \cos \left(t^{\prime}\right)^{2} \xi\left(t^{\prime}\right)^{5}+135 t^{\prime} \cos \left(t^{\prime}\right) \sin \left(t^{\prime}\right) \xi\left(t^{\prime}\right)^{7}\right. \\
+ & \left.-27 t^{\prime} \cos \left(t^{\prime}\right) \sin \left(t^{\prime}\right) \xi\left(t^{\prime}\right)^{5}-\frac{45}{2} \sin \left(t^{\prime}\right)^{2} \xi\left(t^{\prime}\right)^{7}+\frac{27}{2} \sin \left(t^{\prime}\right)^{2} \xi\left(t^{\prime}\right)^{5}\right) \\
+ & \frac{A_{0}^{\prime} B_{0}^{\prime}}{C_{0}^{\prime 4}}\left(135 t^{\prime} \cos \left(t^{\prime}\right)^{2} \xi\left(t^{\prime}\right)^{7}-27 t^{\prime} \cos \left(t^{\prime}\right)^{2} \xi\left(t^{\prime}\right)^{5}-45 \cos \left(t^{\prime}\right) \sin \left(t^{\prime}\right) \xi\left(t^{\prime}\right)^{7}\right. \\
& +405 t^{\prime 2} \cos \left(t^{\prime}\right) \sin \left(t^{\prime}\right) \xi\left(t^{\prime}\right)^{7}-9 \cos \left(t^{\prime}\right) \sin \left(t^{\prime}\right) \xi\left(t^{\prime}\right)^{5}-135 t^{\prime} \sin \left(t^{\prime}\right)^{2} \xi\left(t^{\prime}\right)^{7} \\
& \left.+27 t^{\prime} \sin \left(t^{\prime}\right)^{2} \xi\left(t^{\prime}\right)^{5}\right) \\
+ & \frac{B_{0}^{\prime 2}}{C_{0}^{\prime 4}}\left(-\frac{45}{2} \cos \left(t^{\prime}\right)^{2} \xi\left(t^{\prime}\right)^{7}+\frac{27}{2} \cos \left(t^{\prime}\right)^{2} \xi\left(t^{\prime}\right)^{5}-135 t^{\prime} \cos \left(t^{\prime}\right) \sin \left(t^{\prime}\right) \xi\left(t^{\prime}\right)^{7}\right. \\
& \left.+27 t^{\prime} \cos \left(t^{\prime}\right) \sin \left(t^{\prime}\right) \xi\left(t^{\prime}\right)^{5}-\frac{405}{2} t^{\prime 2} \sin \left(t^{\prime}\right)^{2} \xi\left(t^{\prime}\right)^{7}+18 \sin \left(t^{\prime}\right)^{2} \xi\left(t^{\prime}\right)^{5}\right) \\
+ & O\left(C_{0}^{\prime-5}\right)
\end{aligned}
$$




$$
\begin{aligned}
F_{y}= & +\frac{1}{C_{0}^{\prime 2}} \cdot \frac{9}{2} t^{\prime} \xi\left(t^{\prime}\right)^{3} \\
+ & \frac{A_{0}^{\prime}}{C_{0}^{\prime 3}}\left(\frac{81}{2} t^{\prime 2} \cos \left(t^{\prime}\right) \xi\left(t^{\prime}\right)^{5}-6 \cos \left(t^{\prime}\right) \xi\left(t^{\prime}\right)^{3}-\frac{27}{2} t^{\prime} \sin \left(t^{\prime}\right) \xi\left(t^{\prime}\right)^{5}\right) \\
+ & \frac{B_{0}^{\prime}}{C_{0}^{\prime 3}}\left(\frac{-27}{2} t^{\prime} \cos \left(t^{\prime}\right) \xi\left(t^{\prime}\right)^{5}-\frac{81}{2} t^{\prime 2} \sin \left(t^{\prime}\right) \xi\left(t^{\prime}\right)^{5}+6 \sin \left(t^{\prime}\right) \xi\left(t^{\prime}\right)^{3}\right) \\
+ & \frac{A_{0}^{\prime 2}}{C_{0}^{\prime 4}}\left(\frac{1215}{4} t^{\prime 3} \cos \left(t^{\prime}\right)^{2} \xi\left(t^{\prime}\right)^{7}-81 t^{\prime} \cos \left(t^{\prime}\right)^{2} \xi\left(t^{\prime}\right)^{5}-\frac{405}{2} t^{\prime 2} \cos \left(t^{\prime}\right) \sin \left(t^{\prime}\right) \xi\left(t^{\prime}\right)^{7}\right. \\
& \left.+18 \cos \left(t^{\prime}\right) \sin \left(t^{\prime}\right) \xi\left(t^{\prime}\right)^{5}+\frac{135}{4} t^{\prime} \sin \left(t^{\prime}\right)^{2} \xi\left(t^{\prime}\right)^{7}-\frac{27}{4} t^{\prime} \sin \left(t^{\prime}\right)^{2} \xi\left(t^{\prime}\right)^{5}\right) \\
+ & \frac{A_{0}^{\prime} B_{0}^{\prime}}{C_{0}^{\prime 4}}\left(-\frac{405}{2} t^{\prime 2} \cos \left(t^{\prime}\right)^{2} \xi\left(t^{\prime}\right)^{7}+18 \cos \left(t^{\prime}\right)^{2} \xi\left(t^{\prime}\right)^{5}+\frac{135}{2} t^{\prime} \cos \left(t^{\prime}\right) \sin \left(t^{\prime}\right) \xi\left(t^{\prime}\right)^{7}\right. \\
& -\frac{1215}{2} t^{\prime 3} \cos \left(t^{\prime}\right) \sin \left(t^{\prime}\right) \xi\left(t^{\prime}\right)^{7}+\frac{297}{2} t^{\prime} \cos \left(t^{\prime}\right) \sin \left(t^{\prime}\right) \xi\left(t^{\prime}\right)^{5}+\frac{405}{2} t^{\prime 2} \sin \left(t^{\prime}\right)^{2} \xi\left(t^{\prime}\right)^{7} \\
& \left.-18 \sin \left(t^{\prime}\right)^{2} \xi\left(t^{\prime}\right)^{5}\right) \\
+ & \frac{B_{0}^{\prime 2}}{C_{0}^{\prime 4}}\left(\frac{135}{4} t^{\prime} \cos \left(t^{\prime}\right)^{2} \xi\left(t^{\prime}\right)^{7}-\frac{27}{4} t^{\prime} \cos \left(t^{\prime}\right)^{2} \xi\left(t^{\prime}\right)^{5}+\frac{405}{2} t^{\prime 2} \cos \left(t^{\prime}\right) \sin \left(t^{\prime}\right) \xi\left(t^{\prime}\right)^{7}\right. \\
& \left.-18 \cos \left(t^{\prime}\right) \sin \left(t^{\prime}\right) \xi\left(t^{\prime}\right)^{5}-\frac{1215}{4} t^{\prime 3} \sin \left(t^{\prime}\right)^{2} \xi\left(t^{\prime}\right)^{7}-81 t^{\prime} \sin \left(t^{\prime}\right)^{2} \xi\left(t^{\prime}\right)^{5}\right) \\
+ & O\left(C_{0}^{\prime-5}\right) .
\end{aligned}
$$

Here $\xi\left(t^{\prime}\right)$ is defined by

$$
\xi\left(t^{\prime}\right)=\left(1+\frac{9}{4} t^{\prime 2}\right)^{-1 / 2} .
$$

Equations (27)-(30) can be derived by combining (A1)-(A2) with the equations of motion in the form (14)-(17) and expressing the powers $\sin ^{k}\left(t^{\prime}\right)$ and $\cos ^{k}\left(t^{\prime}\right)$ in terms of $\sin \left(n t^{\prime}\right)$ and $\cos \left(n t^{\prime}\right)$, where the coefficients $a_{i}\left(t^{\prime}\right), b_{i}\left(t^{\prime}\right), c_{i}\left(t^{\prime}\right)$ and $d_{i}\left(t^{\prime}\right)$ with $i=1 \ldots 6$ are

\section{defined}

$$
\begin{aligned}
a_{1}\left(t^{\prime}\right)= & -3 \cos \left(t^{\prime}\right) \xi\left(t^{\prime}\right)^{3}-9 t^{\prime} \sin \left(t^{\prime}\right) \xi\left(t^{\prime}\right)^{3} \\
a_{2}\left(t^{\prime}\right)= & -27 t^{\prime} \cos \left(2 t^{\prime}\right) \xi\left(t^{\prime}\right)^{5}+\frac{9}{2} \sin \left(2 t^{\prime}\right) \xi\left(t^{\prime}\right)^{5}-\frac{81}{2} t^{\prime 2} \sin \left(2 t^{\prime}\right) \xi\left(t^{\prime}\right)^{5} \\
& +\frac{9}{2} \sin \left(2 t^{\prime}\right) \xi\left(t^{\prime}\right)^{3} \\
a_{3}\left(t^{\prime}\right)= & \frac{9}{2} \xi\left(t^{\prime}\right)^{5}+\frac{81}{2} t^{\prime 2} \xi\left(t^{\prime}\right)^{5}-\frac{15}{2} \xi\left(t^{\prime}\right)^{3}+\frac{9}{2} \cos \left(2 t^{\prime}\right) \xi\left(t^{\prime}\right)^{5}
\end{aligned}
$$




$$
\begin{aligned}
& -\frac{81}{2} t^{\prime 2} \cos \left(2 t^{\prime}\right) \xi\left(t^{\prime}\right)^{5}+\frac{9}{2} \cos \left(2 t^{\prime}\right) \xi\left(t^{\prime}\right)^{3}+27 t^{\prime} \sin \left(2 t^{\prime}\right) \xi\left(t^{\prime}\right)^{5} \\
& a_{4}\left(t^{\prime}\right)=-\frac{45}{8} \cos \left(t^{\prime}\right) \xi\left(t^{\prime}\right)^{7}-\frac{405}{8} t^{\prime 2} \cos \left(t^{\prime}\right) \xi\left(t^{\prime}\right)^{7}+\frac{63}{8} \cos \left(t^{\prime}\right) \xi\left(t^{\prime}\right)^{5} \\
& +\frac{45}{8} \cos \left(3 t^{\prime}\right) \xi\left(t^{\prime}\right)^{7} \frac{1215}{8} t^{\prime 2} \cos \left(3 t^{\prime}\right) \xi\left(t^{\prime}\right)^{7}+\frac{81}{8} \cos \left(3 t^{\prime}\right) \xi\left(t^{\prime}\right)^{5} \\
& -\frac{135}{8} t^{\prime} \sin \left(t^{\prime}\right) \xi\left(t^{\prime}\right)^{7}-\frac{1215}{8} t^{\prime 3} \sin \left(t^{\prime}\right) \xi\left(t^{\prime}\right)^{7} \frac{351}{8} t^{\prime} \sin \left(t^{\prime}\right) \xi\left(t^{\prime}\right)^{5} \\
& +\frac{405}{8} t^{\prime} \sin \left(3 t^{\prime}\right) \xi\left(t^{\prime}\right)^{7}-\frac{1215}{8} t^{\prime 3} \sin \left(3 t^{\prime}\right) \xi\left(t^{\prime}\right)^{7}+\frac{243}{8} t^{\prime} \sin \left(3 t^{\prime}\right) \xi\left(t^{\prime}\right)^{5} \\
& a_{5}\left(t^{\prime}\right)=\frac{135}{4} t^{\prime} \cos \left(t^{\prime}\right) \xi\left(t^{\prime}\right)^{7}+\frac{1215}{4} t^{\prime 3} \cos \left(t^{\prime}\right) \xi\left(t^{\prime}\right)^{7}-\frac{351}{4} t^{\prime} \cos \left(t^{\prime}\right) \xi\left(t^{\prime}\right)^{5} \\
& +\frac{405}{4} t^{\prime} \cos \left(3 t^{\prime}\right) \xi\left(t^{\prime}\right)^{7}-\frac{1215}{4} t^{\prime 3} \cos \left(3 t^{\prime}\right) \xi\left(t^{\prime}\right)^{7}+\frac{243}{4} t^{\prime} \cos \left(3 t^{\prime}\right) \xi\left(t^{\prime}\right)^{5} \\
& -\frac{45}{4} \sin \left(t^{\prime}\right) \xi\left(t^{\prime}\right)^{7}-\frac{405}{4} t^{\prime 2} \sin \left(t^{\prime}\right) \xi\left(t^{\prime}\right)^{7}+\frac{63}{4} \sin \left(t^{\prime}\right) \xi\left(t^{\prime}\right)^{5} \\
& -\frac{45}{4} \sin \left(3 t^{\prime}\right) \xi\left(t^{\prime}\right)^{7}+\frac{1215}{4} t^{\prime 2} \sin \left(3 t^{\prime}\right) \xi\left(t^{\prime}\right)^{7}-\frac{81}{4} \sin \left(3 t^{\prime}\right) \xi\left(t^{\prime}\right)^{5} \\
& a_{6}\left(t^{\prime}\right)=-\frac{135}{8} \cos \left(t^{\prime}\right) \xi\left(t^{\prime}\right)^{7}-\frac{1215}{8} t^{\prime 2} \cos \left(t^{\prime}\right) \xi\left(t^{\prime}\right)^{7}+\frac{189}{8} \cos \left(t^{\prime}\right) \xi\left(t^{\prime}\right)^{5} \\
& -\frac{45}{8} \cos \left(3 t^{\prime}\right) \xi\left(t^{\prime}\right)^{7}+\frac{1215}{8} t^{\prime 2} \cos \left(3 t^{\prime}\right) \xi\left(t^{\prime}\right)^{7}-\frac{81}{8} \cos \left(3 t^{\prime}\right) \xi\left(t^{\prime}\right)^{5} \\
& -\frac{405}{8} t^{\prime} \sin \left(t^{\prime}\right) \xi\left(t^{\prime}\right)^{7}-\frac{3645}{8} t^{\prime 3} \sin \left(t^{\prime}\right) \xi\left(t^{\prime}\right)^{7}+\frac{1053}{8} t^{\prime} \sin \left(t^{\prime}\right) \xi\left(t^{\prime}\right)^{5} \\
& -\frac{405}{8} t^{\prime} \sin \left(3 t^{\prime}\right) \xi\left(t^{\prime}\right)^{7}+\frac{1215}{8} t^{\prime 3} \sin \left(3 t^{\prime}\right) \xi\left(t^{\prime}\right)^{7}-\frac{243}{8} t^{\prime} \sin (3 t) \xi\left(t^{\prime}\right)^{5} \\
& b_{1}\left(t^{\prime}\right)=-9 t^{\prime} \cos \left(t^{\prime}\right) \xi\left(t^{\prime}\right)^{3}+3 \sin \left(t^{\prime}\right) \xi\left(t^{\prime}\right)^{3} \\
& b_{2}\left(t^{\prime}\right)=-\frac{9}{2} \xi\left(t^{\prime}\right)^{5}-\frac{81}{2} \xi\left(t^{\prime}\right)^{5}+\frac{15}{2} \xi\left(t^{\prime}\right)^{3}+\frac{9}{2} \cos \left(2 t^{\prime}\right) \xi\left(t^{\prime}\right)^{5} \\
& -\frac{81}{2} t^{\prime 2} \cos \left(2 t^{\prime}\right) \xi\left(t^{\prime}\right)^{5}+\frac{9}{2} \cos \left(2 t^{\prime}\right) \xi\left(t^{\prime}\right)^{3}+27 t^{\prime} \sin \left(2 t^{\prime}\right) \xi\left(t^{\prime}\right)^{5} \\
& b_{3}\left(t^{\prime}\right)=27 t^{\prime} \cos \left(2 t^{\prime}\right) \xi\left(t^{\prime}\right)^{5}-\frac{9}{2} \sin \left(2 t^{\prime}\right) \xi\left(t^{\prime}\right)^{5}+\frac{81}{2} t^{\prime 2} \sin \left(2 t^{\prime}\right) \xi\left(t^{\prime}\right)^{5} \\
& -\frac{9}{2} \sin \left(2 t^{\prime}\right) \xi\left(t^{\prime}\right)^{3} \\
& b_{4}\left(t^{\prime}\right)=-\frac{405}{8} t^{\prime} \cos \left(t^{\prime}\right) \xi\left(t^{\prime}\right)^{7}-\frac{3645}{8} t^{\prime 3} \cos \left(t^{\prime}\right) \xi\left(t^{\prime}\right)^{7}+\frac{105}{8} 3 t^{\prime} \cos \left(t^{\prime}\right) \xi\left(t^{\prime}\right)^{5} \\
& +\frac{405}{8} t^{\prime} \cos \left(3 t^{\prime}\right) \xi\left(t^{\prime}\right)^{7}-\frac{1215}{8} t^{\prime 3} \cos \left(3 t^{\prime}\right) \xi\left(t^{\prime}\right)^{7}+\frac{243}{8} t^{\prime} \cos \left(3 t^{\prime}\right) \xi\left(t^{\prime}\right)^{5} \\
& +\frac{135}{8} \sin \left(t^{\prime}\right) \xi\left(t^{\prime}\right)^{7}+\frac{1215}{8} t^{\prime 2} \sin \left(t^{\prime}\right) \xi\left(t^{\prime}\right)^{7}-\frac{189}{8} \sin \left(t^{\prime}\right) \xi\left(t^{\prime}\right)^{5}
\end{aligned}
$$




$$
\begin{aligned}
& -\frac{45}{8} \sin \left(3 t^{\prime}\right) \xi\left(t^{\prime}\right)^{7}+\frac{1215}{8} t^{\prime 2} \sin \left(3 t^{\prime}\right) \xi\left(t^{\prime}\right)^{7}-\frac{81}{8} \sin \left(3 t^{\prime}\right) \xi\left(t^{\prime}\right)^{5} \\
b_{5}\left(t^{\prime}\right)= & \frac{45}{4} \cos \left(t^{\prime}\right) \xi\left(t^{\prime}\right)^{7}+\frac{405}{4} t^{\prime 2} \cos \left(t^{\prime}\right) \xi\left(t^{\prime}\right)^{7}-\frac{63}{4} \cos \left(t^{\prime}\right) \xi\left(t^{\prime}\right)^{5} \\
& -\frac{45}{4} \cos \left(3 t^{\prime}\right) \xi\left(t^{\prime}\right)^{7}+\frac{1215}{4} t^{\prime 2} \cos \left(3 t^{\prime}\right) \xi\left(t^{\prime}\right)^{7}-\frac{81}{4} \cos \left(3 t^{\prime}\right) \xi\left(t^{\prime}\right)^{5} \\
& +\frac{135}{4} t^{\prime} \sin \left(t^{\prime}\right) \xi\left(t^{\prime}\right)^{7}+\frac{1215}{4} t^{\prime 3} \sin \left(t^{\prime}\right) \xi\left(t^{\prime}\right)^{7}-\frac{351}{4} t^{\prime} \sin \left(t^{\prime}\right) \xi\left(t^{\prime}\right)^{5} \\
& -\frac{405}{4} t^{\prime} \sin \left(3 t^{\prime}\right) \xi\left(t^{\prime}\right)^{7}+\frac{1215}{4} t^{\prime 3} \sin \left(3 t^{\prime}\right) \xi\left(t^{\prime}\right)^{7}-\frac{243}{4} t^{\prime} \sin \left(3 t^{\prime}\right) \xi\left(t^{\prime}\right)^{5} \\
b_{6}\left(t^{\prime}\right)= & -\frac{135}{8} t^{\prime} \cos \left(t^{\prime}\right) \xi\left(t^{\prime}\right)^{7}-\frac{1215}{8} t^{\prime 3} \cos \left(t^{\prime}\right) \xi\left(t^{\prime}\right)^{7}+\frac{351}{8} t^{\prime} \cos \left(t^{\prime}\right) \xi\left(t^{\prime}\right)^{5} \\
& -\frac{405}{8} t^{\prime} \cos \left(3 t^{\prime}\right) \xi\left(t^{\prime}\right)^{7}+\frac{1215}{8} t^{\prime 3} \cos \left(3 t^{\prime}\right) \xi\left(t^{\prime}\right)^{7}-\frac{243}{8} t^{\prime} \cos \left(3 t^{\prime}\right) \xi\left(t^{\prime}\right)^{5} \\
& +\frac{45}{8} \sin \left(t^{\prime}\right) \xi\left(t^{\prime}\right)^{7}+\frac{405}{8} t^{\prime 2} \sin \left(t^{\prime}\right) \xi\left(t^{\prime}\right)^{7}-\frac{63}{8} \sin \left(t^{\prime}\right) \xi\left(t^{\prime}\right)^{5} \\
& +\frac{45}{8} \sin \left(3 t^{\prime}\right) \xi\left(t^{\prime}\right)^{7}-\frac{1215}{8} t^{\prime 2} \sin \left(3 t^{\prime}\right) \xi\left(t^{\prime}\right)^{7}+\frac{81}{8} \sin \left(3 t^{\prime}\right) \xi\left(t^{\prime}\right)^{5}
\end{aligned}
$$

$$
\begin{aligned}
c_{1}\left(t^{\prime}\right)= & 9 t^{\prime} \xi\left(t^{\prime}\right)^{3} \\
c_{2}\left(t^{\prime}\right)= & 81 t^{\prime 2} \cos \left(t^{\prime}\right) \xi\left(t^{\prime}\right)^{5}-12 \cos \left(t^{\prime}\right) \xi\left(t^{\prime}\right)^{3}-27 t^{\prime} \sin \left(t^{\prime}\right) \xi\left(t^{\prime}\right)^{5} \\
c_{3}\left(t^{\prime}\right)= & -27 t^{\prime} \cos \left(t^{\prime}\right) \xi\left(t^{\prime}\right)^{5}-81 t^{\prime 2} \sin \left(t^{\prime}\right) \xi\left(t^{\prime}\right)^{5}+12 \sin \left(t^{\prime}\right) \xi\left(t^{\prime}\right)^{3} \\
c_{4}\left(t^{\prime}\right)= & \frac{135}{4} t^{\prime} \xi\left(t^{\prime}\right)^{7}+\frac{1215}{4} t^{\prime 3} \xi\left(t^{\prime}\right)^{7}-\frac{351}{4} t^{\prime} \xi\left(t^{\prime}\right)^{5}-\frac{135}{4} t^{\prime} \cos \left(2 t^{\prime}\right) \xi\left(t^{\prime}\right)^{7} \\
& +\frac{1215}{4} t^{\prime 3} \cos \left(2 t^{\prime}\right) \xi\left(t^{\prime}\right)^{7}-\frac{297}{4} t^{\prime} \cos \left(2 t^{\prime}\right) \xi\left(t^{\prime}\right)^{5}-\frac{405}{2} t^{\prime 2} \sin \left(2 t^{\prime}\right) \xi\left(t^{\prime}\right)^{7} \\
& +18 \sin \left(2 t^{\prime}\right) \xi\left(t^{\prime}\right)^{5} \\
c_{5}\left(t^{\prime}\right)= & -405 t^{\prime 2} \cos \left(2 t^{\prime}\right) \xi\left(t^{\prime}\right)^{7}+36 \cos \left(2 t^{\prime}\right) \xi\left(t^{\prime}\right)^{5}+\frac{135}{2} t^{\prime} \sin \left(2 t^{\prime}\right) \xi\left(t^{\prime}\right)^{7} \\
& -\frac{1215}{2} t^{\prime 3} \sin \left(2 t^{\prime}\right) \xi\left(t^{\prime}\right)^{7}+\frac{297}{2} t^{\prime} \sin \left(2 t^{\prime}\right) \xi\left(t^{\prime}\right)^{5} \\
& \frac{135}{4} t^{\prime} \xi\left(t^{\prime}\right)^{7}+\frac{1215}{4} t^{\prime 3} \xi\left(t^{\prime}\right)^{7}-\frac{351}{4} t^{\prime} \xi\left(t^{\prime}\right)^{5}+\frac{135}{4} t^{\prime} \cos \left(2 t^{\prime}\right) \xi\left(t^{\prime}\right)^{7} \\
& -\frac{1215}{4} t^{\prime 3} \cos \left(2 t^{\prime}\right) \xi\left(t^{\prime}\right)^{7}+\frac{297}{4} t^{\prime} \cos \left(2 t^{\prime}\right) \xi\left(t^{\prime}\right)^{5}+\frac{405}{2} t^{\prime 2} \sin \left(2 t^{\prime}\right) \xi\left(t^{\prime}\right)^{7} \\
c_{6}\left(t^{\prime}\right)= & -18 \sin \left(2 t^{\prime}\right) \xi\left(t^{\prime}\right)^{5}
\end{aligned}
$$




$$
\begin{aligned}
& d_{1}\left(t^{\prime}\right)= 6 \xi\left(t^{\prime}\right) \\
& d_{2}\left(t^{\prime}\right)= 54 t^{\prime} \cos (t) \xi\left(t^{\prime}\right)^{5}+\frac{243}{2} t^{\prime 3} \cos (t) \xi\left(t^{\prime}\right)^{5}-18 t^{\prime} \cos (t) \xi\left(t^{\prime}\right)^{3} \\
&-18 \sin (t) \xi\left(t^{\prime}\right)^{5}-\frac{81}{2} t^{\prime 2} \sin (t) \xi\left(t^{\prime}\right)^{5}+6 \sin (t) \xi\left(t^{\prime}\right)^{3} \\
& d_{3}\left(t^{\prime}\right)=-18 \cos (t) \xi\left(t^{\prime}\right)^{5}-\frac{81}{2} t^{\prime 2} \cos (t) \xi\left(t^{\prime}\right)^{5}+6 \cos (t) \xi\left(t^{\prime}\right)^{3} \\
&-54 t^{\prime} \sin (t) \xi\left(t^{\prime}\right)^{5}-\frac{243}{2} t^{\prime 3} \sin (t) \xi\left(t^{\prime}\right)^{5}+18 t^{\prime} \sin (t) \xi\left(t^{\prime}\right)^{3} \\
&\left.45 t^{\prime}\right)^{7}+\frac{2025}{8} t^{\prime 2} \xi\left(t^{\prime}\right)^{7}+\frac{3645}{8} t^{\prime 4} \xi\left(t^{\prime}\right)^{7}-\frac{63}{2} \xi\left(t^{\prime}\right)^{5}-\frac{1053}{8} t^{\prime 2} \xi\left(t^{\prime}\right)^{5} \\
&-\frac{45}{2} \cos \left(2 t^{\prime}\right) \xi\left(t^{\prime}\right)^{7}+\frac{1215}{8} t^{\prime 2} \cos \left(2 t^{\prime}\right) \xi\left(t^{\prime}\right)^{7}+\frac{3645}{8} t^{\prime 4} \cos \left(2 t^{\prime}\right) \xi\left(t^{\prime}\right)^{7} \\
&-\frac{9}{2} \cos \left(2 t^{\prime}\right) \xi\left(t^{\prime}\right)^{5}-\frac{891}{8} t^{\prime 2} \cos \left(2 t^{\prime}\right) \xi\left(t^{\prime}\right)^{5}-135 t^{\prime} \sin \left(2 t^{\prime}\right) \xi\left(t^{\prime}\right)^{7} \\
&-\frac{1215}{4} t^{\prime 3} \sin \left(2 t^{\prime}\right) \xi\left(t^{\prime}\right)^{7}+54 t^{\prime} \sin \left(2 t^{\prime}\right) \xi\left(t^{\prime}\right)^{5} \\
&-270 t^{\prime} \cos \left(2 t^{\prime}\right) \xi\left(t^{\prime}\right)^{7}-\frac{1215}{2} t^{\prime 3} \cos \left(2 t^{\prime}\right) \xi\left(t^{\prime}\right)^{7}+108 t^{\prime} \cos \left(2 t^{\prime}\right) \xi\left(t^{\prime}\right)^{5} \\
&+45 \sin \left(2 t^{\prime}\right) \xi\left(t^{\prime}\right)^{7}-\frac{1215}{4} t^{\prime 2} \sin \left(2 t^{\prime}\right) \xi\left(t^{\prime}\right)^{7}-\frac{3645}{4} t^{\prime 4} \sin \left(2 t^{\prime}\right) \xi\left(t^{\prime}\right)^{7} \\
&+9 \sin \left(2 t^{\prime}\right) \xi\left(t^{\prime}\right)^{5}+\frac{891}{4} t^{\prime 2} \sin \left(2 t^{\prime}\right) \xi\left(t^{\prime}\right)^{5} \\
&+\frac{45}{2} \xi\left(t^{\prime}\right)^{7}+\frac{2025}{8} t^{\prime 2} \xi\left(t^{\prime}\right)^{7}+\frac{3645}{8} t^{\prime 4} \xi\left(t^{\prime}\right)^{7}-\frac{63}{2} \xi\left(t^{\prime}\right)^{5}-\frac{1053}{8} t^{\prime 2} \xi\left(t^{\prime}\right)^{5} \\
& d_{5}\left(t^{\prime}\right)= \frac{9}{2} \cos \left(2 t^{\prime}\right) \xi\left(t^{\prime}\right)^{5}+\frac{891}{8} t^{\prime 2} \cos \left(2 t^{\prime}\right) \xi\left(t^{\prime}\right)^{5}+135 t^{\prime} \sin \left(2 t^{\prime}\right) \xi\left(t^{\prime}\right)^{7} \\
&+\frac{1215}{4} t^{\prime 3} \sin \left(2 t^{\prime}\right) \xi\left(t^{\prime}\right)^{7}-54 t^{\prime} \sin \left(2 t^{\prime}\right) \xi\left(t^{\prime}\right)^{5} \cdot \\
& d_{6}\left(t^{\prime}\right)=\frac{1215}{8} t^{\prime 2} \cos \left(2 t^{\prime}\right) \xi\left(t^{\prime}\right)^{7}-\frac{3645}{8} t^{\prime 4} \cos \left(2 t^{\prime}\right) \xi\left(t^{\prime}\right)^{7} \\
&=
\end{aligned}
$$

The integration of the coefficients $a_{i}\left(t^{\prime}\right), b_{i}\left(t^{\prime}\right), c_{i}\left(t^{\prime}\right)$ and $d_{i}\left(t^{\prime}\right)$ over the time domain from $-\infty$ to $+\infty$ can be done analytically, where the more complex terms can be expressed in terms of Bessel $K$ or Meijer $G$ functions. The resulting numerical values are given in Table 4, where we used the definitions

$$
\begin{aligned}
\mathcal{A}_{i} & =\int_{-\infty}^{+\infty} a_{i}\left(t^{\prime}\right) d t^{\prime}, & \mathcal{B}_{i} & =\int_{-\infty}^{+\infty} b_{i}\left(t^{\prime}\right) d t^{\prime}, \\
\mathcal{C}_{i} & =\int_{-\infty}^{+\infty} c_{i}\left(t^{\prime}\right) d t^{\prime}, & \mathcal{D}_{i} & =\int_{-\infty}^{+\infty} d_{i}\left(t^{\prime}\right) d t^{\prime} .
\end{aligned}
$$


Only the term $\left(1+9 t^{2} / 4\right)^{-1 / 2}$ is divergent. The divergence is an effect of the neglect of curvature and the term has to be handled separately (see section 2.3 and appendix B).

Please, insert table 4 Here.

\section{B. The divergent term $\mathcal{D}_{1}$}

The $\mathcal{D}_{1}$ term describes a shift in the azimuthal direction and can be written in the form

$$
\mathcal{D}_{1}(y)=4\left[\operatorname{arcsinh}\left(\frac{y_{\text {crit }}}{x}\right)-\operatorname{arcsinh}\left(\frac{y}{x}\right)\right]
$$

where $x \approx x_{0}$ and $y \approx y_{0}-3 / 2 x t$ are assumed for simplicity. Figure 12 compares the azimuthal shift term $\mathcal{D}_{1}(y) x^{-2}$ with the azimuthal wavelength $\lambda_{y}=3 \pi x$. The value $y_{\text {crit }}$ is set to zero, as an approximation, because the shift affects strongest in the regions of strong perturbations close to the moon position. After 100 wavelengths the shift is still smaller than $5 \%$ of a wavelength and is a slowly growing function of $y$. This justifies the neglect of $\mathcal{D}_{1}$ as a small value, as it is done in earlier papers (e.g. Showalter et al. (1986)).

Please, insert figure 12 Here.

\section{Order $x_{0}^{-4}$ streamline equations and related streamline density parameters}

Including order $x_{0}^{-4}$ terms the streamline equations can be extended to the form

$$
\begin{aligned}
x_{p}\left(x_{0}, y_{0}, t\right)= & (47)+\operatorname{sign}\left(x_{0}\right)\left\{\frac{\mathcal{C}_{5}}{x_{0}{ }^{4}} e_{0}^{2} \cos \left(\tau-\frac{2 y_{0}}{3 x_{0}}\right) \sin \left(\tau-\frac{2 y_{0}}{3 x_{0}}\right)\right. \\
& +\left[\frac{\mathcal{A}_{4}}{x_{0}{ }^{4}} e_{0}^{2} \sin \left(\tau-\frac{2 y_{0}}{3 x_{0}}\right)^{2}+\frac{\mathcal{A}_{6}}{x_{0}{ }^{4}} e_{0}^{2} \cos \left(\tau-\frac{2 y_{0}}{3 x_{0}}\right)^{2}\right] \sin \left(t-\frac{2 y_{0}}{3 x_{0}}\right) \\
& \left.+\frac{\mathcal{B}_{5}}{x_{0}{ }^{4}} e_{0}^{2} \cos \left(\tau-\frac{2 y_{0}}{3 x_{0}}\right) \sin \left(\tau-\frac{2 y_{0}}{3 x_{0}}\right) \cos \left(t-\frac{2 y_{0}}{3 x_{0}}\right)\right\} \\
y_{p}\left(x_{0}, y_{0}, t\right)= & (48)+\operatorname{sign}\left(x_{0}\right)\left\{\frac{\mathcal{D}_{4}}{x_{0} 4} e_{0}^{2} \sin \left(\tau-\frac{2 y_{0}}{3 x_{0}}\right)^{2}+\frac{\mathcal{D}_{6}}{x_{0}{ }^{4}} e_{0}^{2} \cos \left(\tau-\frac{2 y_{0}}{3 x_{0}}\right)^{2}\right.
\end{aligned}
$$




$$
\begin{aligned}
& +\left[y_{0}-\frac{3}{2} x_{0} t\right] \frac{\mathcal{C}_{5}}{x_{0}{ }^{5}} e_{0}^{2} \cos \left(\tau-\frac{2 y_{0}}{3 x_{0}}\right) \sin \left(\tau-\frac{2 y_{0}}{3 x_{0}}\right) \\
& +\left[\frac{2 \mathcal{A}_{4}}{x_{0}{ }^{4}} e_{0}^{2} \sin \left(\tau-\frac{2 y_{0}}{3 x_{0}}\right)^{2}+\frac{2 \mathcal{A}_{6}}{x_{0}{ }^{4}} e_{0}^{2} \cos \left(\tau-\frac{2 y_{0}}{3 x_{0}}\right)^{2}\right] \cos \left(t-\frac{2 y_{0}}{3 x_{0}}\right) \\
& \left.-\frac{2 \mathcal{B}_{5}}{x_{0}{ }^{4}} e_{0}^{2} \cos \left(\tau-\frac{2 y_{0}}{3 x_{0}}\right) \sin \left(\tau-\frac{2 y_{0}}{3 x_{0}}\right) \sin \left(t-\frac{2 y_{0}}{3 x_{0}}\right)\right\}
\end{aligned}
$$

The parameters of the streamline density become

$$
\begin{aligned}
p= & \frac{p_{1} e s}{X^{4}} \sin \left(\frac{2 Y}{3 X}-\tau\right)+\frac{p_{2} e^{2} s}{X^{5}} \cos \left(\frac{2 Y}{3 X}-\tau\right) \sin \left(\frac{2 Y}{3 X}-\tau\right)+O\left(X^{-6}\right) \\
= & \frac{p_{1} e s}{X^{4}} \sin \left(\frac{2 Y}{3 X}-\tau\right)+O\left(X^{-6}\right) \\
q= & q_{0}\left[1+\frac{q_{1} e}{X} \cos \left(\frac{2 Y}{3 X}+t-\tau\right)+\frac{q_{2} e^{2}}{X^{2}} \cos \left(\frac{2 Y}{3 X}+t-\tau\right)^{2}\right. \\
& \left.+\frac{q_{3} e^{2}}{X^{2}} \sin \left(\frac{2 Y}{3 X}+t-\tau\right)^{2}\right]+q_{0} \cdot O\left(X^{-3}\right) \\
\gamma= & \frac{\gamma_{1} e}{X} \sin \left(\frac{2 Y}{3 X}+t-\tau\right)+\frac{\gamma_{2} e^{2}}{X^{2}} \cos \left(\frac{2 Y}{3 X}+t-\tau\right) \sin \left(\frac{2 Y}{3 X}+t-\tau\right) \\
& +O\left(X^{-3}\right)
\end{aligned}
$$

using the following definitions:

$$
\begin{aligned}
& p_{1}=-2 \mathcal{C}_{2}+\frac{2 \mathcal{D}_{3}}{3} \approx 14.6845 \\
& p_{2}=3 \mathcal{C}_{5}+\frac{4 \mathcal{D}_{4}}{3}-\frac{4 \mathcal{D}_{6}}{3}=0 \\
& q_{1}=-\frac{\mathcal{A}_{3}+\mathcal{B}_{2}}{\mathcal{A}_{1}} \approx-11.4176 \\
& q_{2}=\frac{\mathcal{A}_{6}+\mathcal{B}_{5}}{\mathcal{A}_{1}} \approx-11.0663 \\
& q_{3}=\frac{\left(\mathcal{A}_{3}+\mathcal{B}_{2}\right)^{2}}{2 \mathcal{A}_{1}^{2}}+\frac{\mathcal{A}_{4}-\mathcal{B}_{5}}{\mathcal{A}_{1}} \approx-1.8572 \\
& \gamma_{1}=-q_{1} \approx 11.4176 \\
& \gamma_{2}=2\left(q_{3}-q_{2}\right) \approx 18.4182
\end{aligned}
$$


D. Tables of image parameters

Please, insert table 5 Here.

Please, insert table 6 Here. 


\section{REFERENCES}

Borderies, N., Goldreich, P., Tremaine, S., Sep. 1982. Sharp edges of planetary rings. Nature 299, 209-211.

Borderies, N., Goldreich, P., Tremaine, S., Aug. 1989. The formation of sharp edges in planetary rings by nearby satellites. Icarus $80,344-360$.

Bosh, A. S., Olkin, C. B., French, R. G., Nicholson, P. D., May 2002. Saturn's F Ring: Kinematics and Particle Sizes from Stellar Occultation Studies. Icarus 157, 57-75.

Burns, J. A., Hedman, M. M., Tiscareno, M. S., Nicholson, P. D., Streetman, B. J., Colwell, J. E., Showalter, M. R., Murray, C. D., Cuzzi, J. N., Porco, C. C., Cassini ISS Team, Aug. 2005. Morphology, Movements and Models of Ringlets in Saturn's Encke Gap. In: Bulletin of the American Astronomical Society. Vol. 37 of Bulletin of the American Astronomical Society. p. 766.

Colwell, J. E., Esposito, L. W., Sremčević, M., Apr. 2006. Self-gravity wakes in Saturn's A ring measured by stellar occultations from Cassini. Geophysical Research Letters 33, $7201-+$.

Cuzzi, J. N., Scargle, J. D., May 1985. Wavy edges suggest moonlet in Encke's gap. Astrophysical Journal 292, 276-290.

Goldreich, P., Tremaine, S., Oct. 1980. Disk-satellite interactions. Astrophysical Journal $241,425-441$.

Hahn, J. M., Aug. 2007. The Secular Evolution of a Close Ring-Satellite System: The Excitation of Spiral Bending Waves at a Nearby Gap Edge. Astrophysical Journal 665, 856-865. 
Hedman, M. M., Burns, J. A., Tiscareno, M. S., Porco, C. C., Oct. 2007. The Heliotropic Rings of Saturn. In: AAS/Division for Planetary Sciences Meeting Abstracts. Vol. 39 of AAS/Division for Planetary Sciences Meeting Abstracts. p. 10.09.

Henon, M., Sep. 1981. A simple model of Saturn's rings. Nature 293, 33-35.

Henon, M., Petit, J.-M., Jan. 1986. Series expansion for encounter-type solutions of Hill's problem. Celestial Mechanics 38, 67-100.

Hertzsch, J., Scholl, H., Spahn, F., Katzorke, I., Apr. 1997. Simulation of collisions in planetary rings. Astronomy and Astrophysics 320, 319-324.

Hill, G., 1878. Researches in the lunar theory. Am. J. Math. 1, 5-26.

Horn, L. J., Showalter, M. R., Russell, C. T., Dec. 1996. Detection and Behavior of Pan Wakes in Saturn's A Ring. Icarus 124, 663-676.

Jacobson, R. A., Spitale, J., Porco, C. C., Beurle, K., Cooper, N. J., Evans, M. W., Murray, C. D., Jan. 2008. Revised Orbits of Saturn's Small Inner Satellites. Astronomical Journal 135, 261-263.

Julian, W. H., Toomre, A., Dec. 1966. Non-Axisymmetric Responses of Differentially Rotating Disks of Stars. Astrophysical Journal 146, 810-+.

Lewis, M. C., Stewart, G. R., Dec. 2000. Collisional Dynamics of Perturbed Planetary Rings. I. Astronomical Journal 120, 3295-3310.

Lewis, M. C., Stewart, G. R., Sep. 2006. Simulating the Keeler Gap in Saturn's Rings: Wake and Edge Dynamics. In: Bulletin of the American Astronomical Society. Vol. 38 of Bulletin of the American Astronomical Society. pp. 560-+.

Lissauer, J. J., Shu, F. H., Cuzzi, J. N., Aug. 1981. Moonlets in Saturn's rings. Nature 292, $707-711$. 
Moons, M., Delhaise, F., Depaepe, E., 1988. Elliptical Hill's problem (large and small impact parameters). Celestial Mechanics 43, 349-359.

Petit, J.-M., Henon, M., Jun. 1988. A numerical simulation of planetary rings. III-- Mass segregation, ring confinement, and gap formation. Astronomy and Astrophysics 199, $343-356$.

Porco, C. C., May 2005. S/2005 S 1. IAU Circ. 8524, 1-+.

Porco, C. C., Baker, E., Barbara, J., Beurle, K., Brahic, A., Burns, J. A., Charnoz, S., Cooper, N., Dawson, D. D., Del Genio, A. D., Denk, T., Dones, L., Dyudina, U., Evans, M. W., Giese, B., Grazier, K., Helfenstein, P., Ingersoll, A. P., Jacobson, R. A., Johnson, T. V., McEwen, A., Murray, C. D., Neukum, G., Owen, W. M., Perry, J., Roatsch, T., Spitale, J., Squyres, S., Thomas, P., Tiscareno, M., Turtle, E., Vasavada, A. R., Veverka, J., Wagner, R., West, R., Feb. 2005. Cassini Imaging Science: Initial Results on Saturn's Rings and Small Satellites. Science 307, $1226-1236$.

Porco, C. C., Thomas, P. C., Weiss, J. W., Richardson, D. C., Dec. 2007. Saturn's Small Inner Satellites: Clues to Their Origins. Science 318, 1602-.

Porco, C. C., West, R. A., Squyres, S., McEwen, A., Thomas, P., Murray, C. D., Delgenio, A., Ingersoll, A. P., Johnson, T. V., Neukum, G., Veverka, J., Dones, L., Brahic, A., Burns, J. A., Haemmerle, V., Knowles, B., Dawson, D., Roatsch, T., Beurle, K., Owen, W., Dec. 2004. Cassini Imaging Science: Instrument Characteristics And Anticipated Scientific Investigations At Saturn. Space Science Reviews 115, 363-497.

Salo, H., Oct. 1992. Gravitational wakes in Saturn's rings. Nature 359, 619-621.

Salo, H., Oct. 1995. Simulations of dense planetary rings. III. Self-gravitating identical particles. Icarus 117, 287-312. 
Seiß, M., Spahn, F., Sremčević, M., Salo, H., Jun. 2005. Structures induced by small moonlets in Saturn's rings: Implications for the Cassini Mission. Geophysical Research Letters 32, 11205-+.

Showalter, M. R., Jun. 1991. Visual detection of 1981S13, Saturn's eighteenth satellite, and its role in the Encke gap. Nature 351, 709-713.

Showalter, M. R., Cuzzi, J. N., Marouf, E. A., Esposito, L. W., May 1986. Satellite 'wakes' and the orbit of the Encke Gap moonlet. Icarus 66, 297-323.

Spahn, F., Greiner, J., Schwarz, U., Nov. 1992a. Moonlets in Saturn's rings. Advances in Space Research 12, 141-147.

Spahn, F., Saar, A., Schmidt, S., Schwarz, U., Nov. 1992b. The influence of various moonlets on the optical depth profile in planetary rings. Icarus 100, 143-153.

Spahn, F., Scholl, H., Hertzsch, J., Oct. 1994. Structures in planetary rings caused by embedded moonlets. Icarus 111, 514-535.

Spahn, F., Sponholz, H., Jun. 1989. Existence of moonlets in Saturn's rings inferred from the optical depth profile. Nature 339, 607-608.

Spahn, F., Sremčević, M., Jun. 2000. Density patterns induced by small moonlets in Saturn's rings? Astronomy and Astrophysics 358, 368-372.

Spahn, F., Wiebicke, H.-J., Jan. 1989. Long-term gravitational influence of moonlets in planetary rings. Icarus $77,124-134$.

Sremčević, M., Schmidt, J., Salo, H., Seiß, M., Spahn, F., Albers, N., Oct. 2007. A belt of moonlets in Saturn's A ring. Nature 449, 1019-1021.

Sremčević, M., Spahn, F., Duschl, W. J., Dec. 2002. Density structures in perturbed thin cold discs. Monthly Notices Royal Astron. Soc. 337, 1139-1152. 
Stewart, G. R., Dec. 1991. Nonlinear satellite wakes in planetary rings. I - Phase-space kinematics. Icarus 94, 436-450.

Thomson, F. S., Marouf, E. A., Tyler, G. L., French, R. G., Rappoport, N. J., Dec. 2007. Periodic microstructure in Saturn's rings A and B. Geophysical Research Letters 34, $24203-+$.

Tiscareno, M. S., Burns, J. A., Hedman, M. M., Porco, C. C., Mar. 2008. The Population of Propellers in Saturn's a Ring. Astronomical Journal 135, 1083-1091.

Tiscareno, M. S., Burns, J. A., Hedman, M. M., Porco, C. C., Weiss, J. W., Dones, L., Richardson, D. C., Murray, C. D., Mar. 2006. 100-metre-diameter moonlets in Saturn's A ring from observations of 'propeller' structures. Nature 440, 648-650.

Tiscareno, M. S., Burns, J. A., Hedman, M. M., Spitale, J. N., Porco, C. C., Murray, C. D., Cassini Imaging Team, Aug. 2005. Wavy Edges and Other Disturbances in Saturn's Encke and Keeler Gaps. In: Bulletin of the American Astronomical Society. Vol. 37 of Bulletin of the American Astronomical Society. pp. 767-+.

Weiss, J. W., Porco, C. C., Tiscareno, M. S., Jul. 2009. Ring Edge Waves and the Masses of Nearby Satellites. Astronomical Journal 138, 272-286.

Weiss, J. W., Porco, C. C., Tiscareno, M. S., Burns, J. A., Dones, L., Aug. 2005. The Determination of the Masses of Ring-Embedded Moons from their Effects on Nearby Ring Particles. In: Bulletin of the American Astronomical Society. Vol. 37 of Bulletin of the American Astronomical Society. pp. 767-+.

Zebker, H. A., Marouf, E. A., Tyler, G. L., Dec. 1985. Saturn's rings - Particle size distributions for thin layer model. Icarus $64,531-548$.

This manuscript was prepared with the AAS LATEX macros v5.2. 

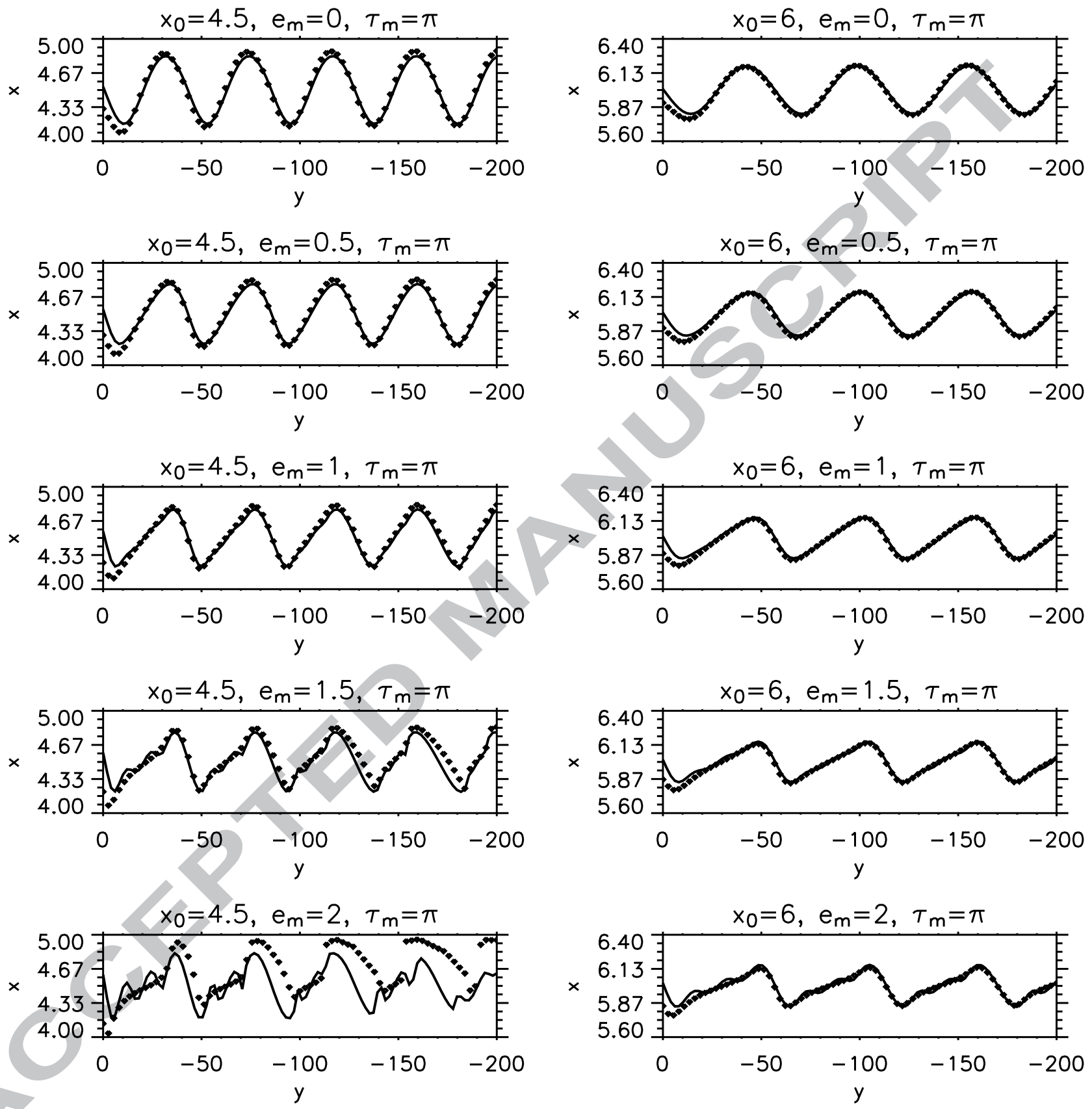

Fig. 1.- Comparison between analytical and numerical results for a streamline. The numerical solution is denoted by dots and the analytical solution to order $C^{-4}$ by a line. The initial values $x_{0}=4.5$ (left panels) and $x_{0}=6.0$ (right panels) as well as $t=0$ and $\tau_{m}=\pi$ are kept constant, while $e_{m}$ is varied. The initial particle eccentricity is zero $e_{p, 0}=0$, so that $e_{0}=e_{m}$ 

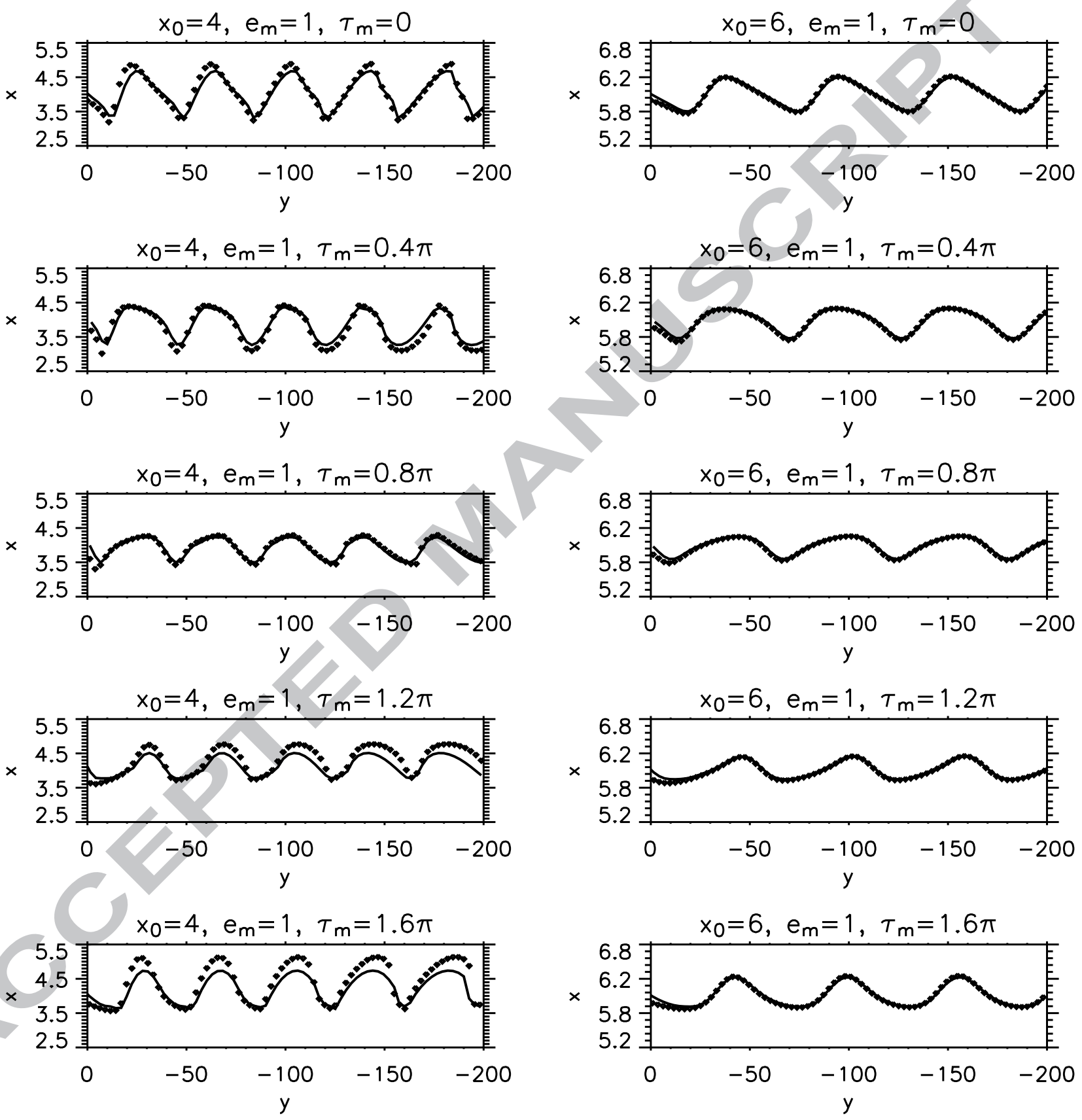

Fig. 2.- Same as figure 1, except that $e_{m}=1$ is chosen constant and $\tau_{m}$ is varied. 

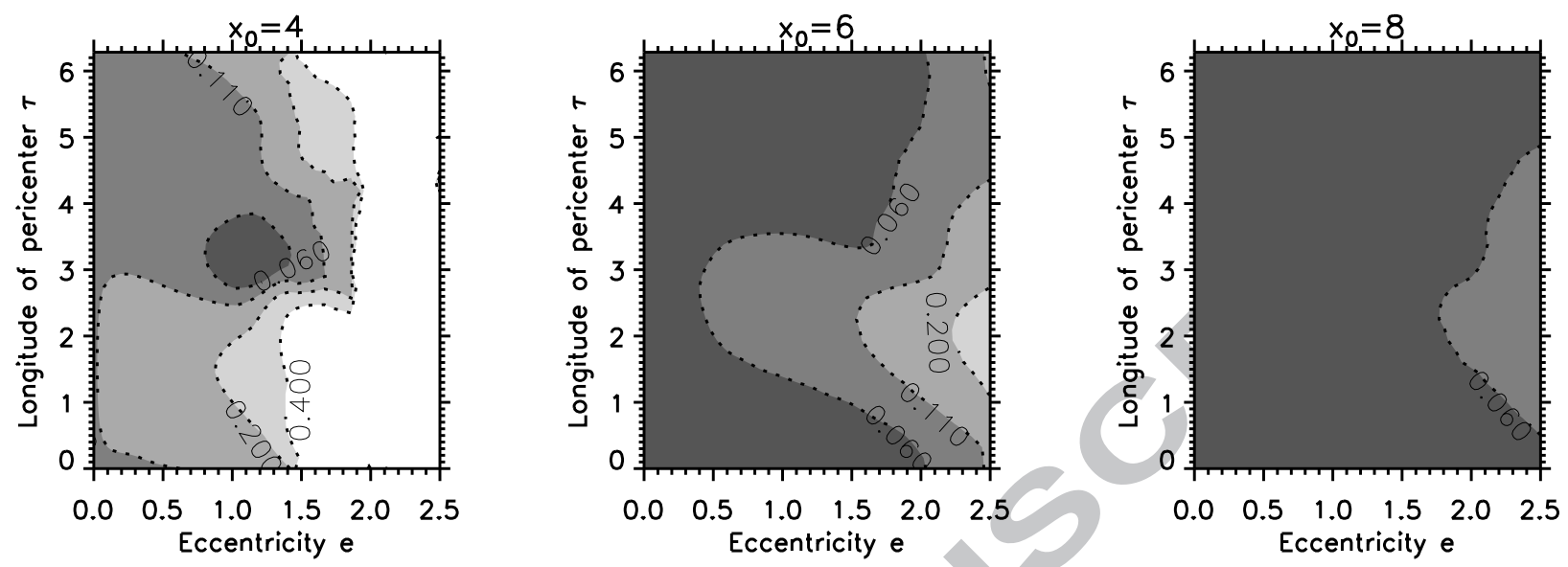

Fig. 3.- Deviation of the analytical model (4th order solution) from the exact solution for impact parameters $x_{0}=4,6$ and 8 as defined by equation (49). The deviation is measured over the second and third period.

Table 1: Parameters fitted from the inner Keeler gap edge

\begin{tabular}{cccccc}
\hline \hline Image name & Var, $\mathrm{km}^{2}$ & $h, \mathrm{~km}$ & $x_{0}$ & $e$ & $\tau-t+t_{\text {epoch }}$ \\
\hline $\mathrm{N} 1493722512$ & 0.02 & $5.16_{+0.03}^{-0.03}$ & $-3.87_{+0.02}^{-0.02}$ & $0.31_{+0.05}^{-0.05}$ & $4.27_{+0.06}^{-0.06}$ \\
$\mathrm{~N} 1493722609$ & 0.05 & $5.15_{+0.04}^{-0.04}$ & $-3.87_{+0.02}^{-0.02}$ & $0.40_{+0.06}^{-0.06}$ & $3.98_{+0.13}^{-0.06}$ \\
$\mathrm{~N} 1536484175$ & 0.20 & $5.48_{+0.12}^{-0.10}$ & $-4.12_{+0.07}^{-0.07}$ & $1.29_{+0.09}^{-0.07}$ & $3.14_{+0.19}^{-0.25}$ \\
$\mathrm{~N} 1540685777$ & 0.08 & $5.28_{+0.06}^{-0.06}$ & $-3.94_{+0.05}^{-0.05}$ & $1.18_{+0.05}^{-0.05}$ & $3.55_{+0.06}^{-0.00}$ \\
$\mathrm{~N} 1547691181$ & 0.34 & $5.27_{+0.10}^{-0.08}$ & $-4.11_{+0.08}^{-0.10}$ & $0.89_{+0.10}^{-0.08}$ & $0.20_{+0.19}^{-0.32}$ \\
$\mathrm{~N} 1547691776$ & 0.36 & $5.19_{+0.10}^{-0.10}$ & $-4.20_{+0.09}^{-0.09}$ & $0.93_{+0.10}^{-0.10}$ & $0.19_{+0.25}^{-0.32}$ \\
$\mathrm{~N} 1553466638$ & 0.64 & $5.00_{+0.13}^{-0.12}$ & $-4.34_{+0.11}^{-0.13}$ & $1.25_{+0.13}^{-0.14}$ & $6.16_{+0.32}^{-0.38}$ \\
$\mathrm{~N} 1553469038$ & 1.09 & $5.11_{+0.16}^{-0.18}$ & $-4.32_{+0.17}^{-0.18}$ & $1.00_{+0.20}^{-0.21}$ & $5.78_{+0.76}^{-0.32}$ \\
\hline
\end{tabular}

Note. - The parameters $x_{0}, e$ and $\tau$ denote the scaled (dimensionless) impact parameter, initial effective eccentricity and initial effective longitude of pericenter, respectively. Var measures the variance between the data and the best fit and $h$ labels the fitted Hill radius of perturbing moon. 

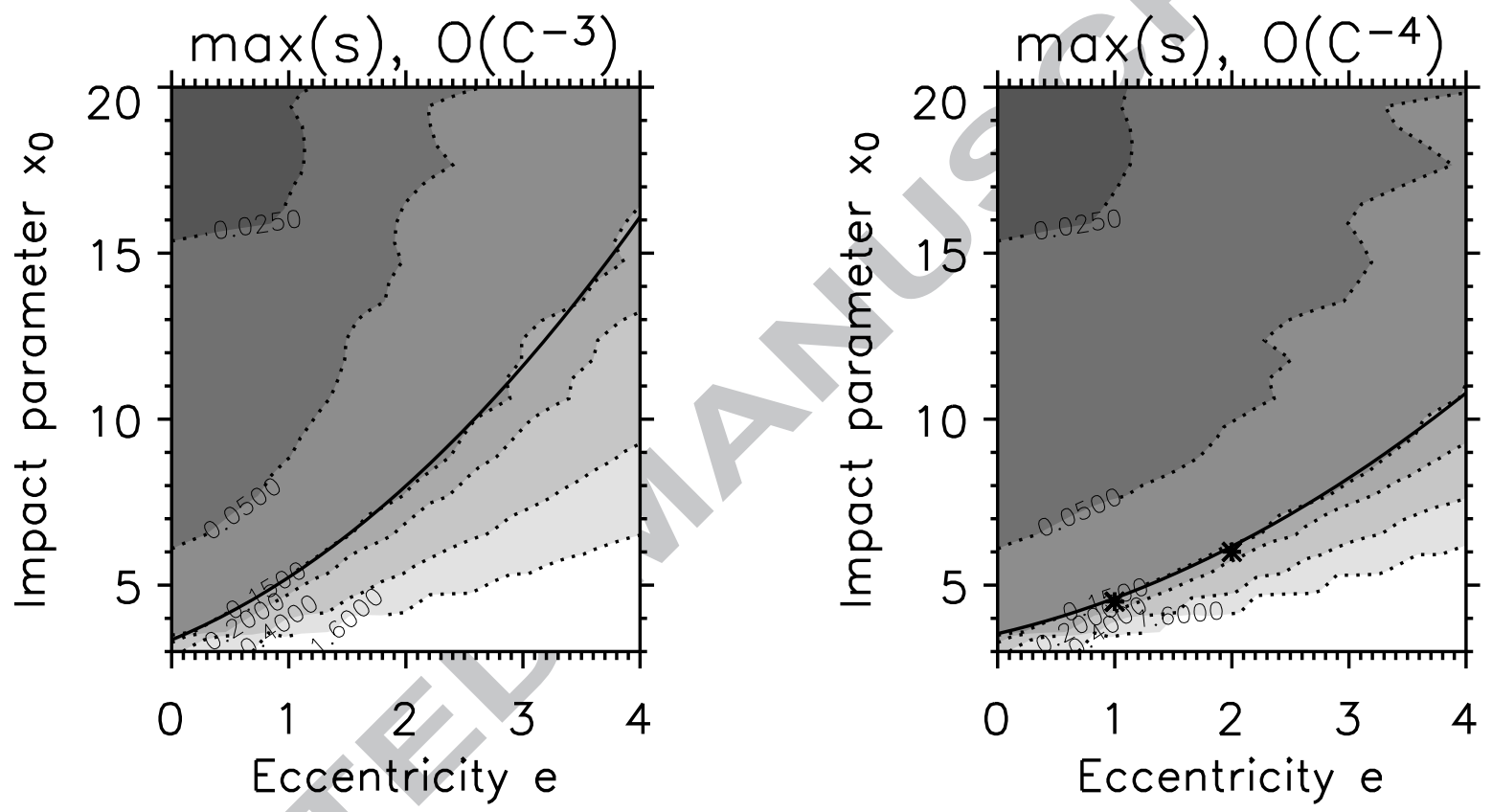

Fig. 4. - Maximal error of the analytical model $O\left(x_{0}^{-3}\right)$ (left panel) and $O\left(x_{0}^{-4}\right)$ (right panel), depending on the moonlet eccentricity and impact parameter. The solid line represents the estimate for the applicability of the model (For $O\left(x_{0}^{-3}\right): x_{0}>x_{0, \text { crit }}=0.43 \cdot e^{2}+$ $1.4 \cdot e+3.5$, and for $\left.O\left(x_{0}^{-4}\right): x_{0}>x_{0, \text { crit }}=0.25 \cdot e^{2}+0.8 \cdot e+3.5\right)$. The stars in the right panel correspond to the model applicability estimate based on the figures 1 and 2 . 

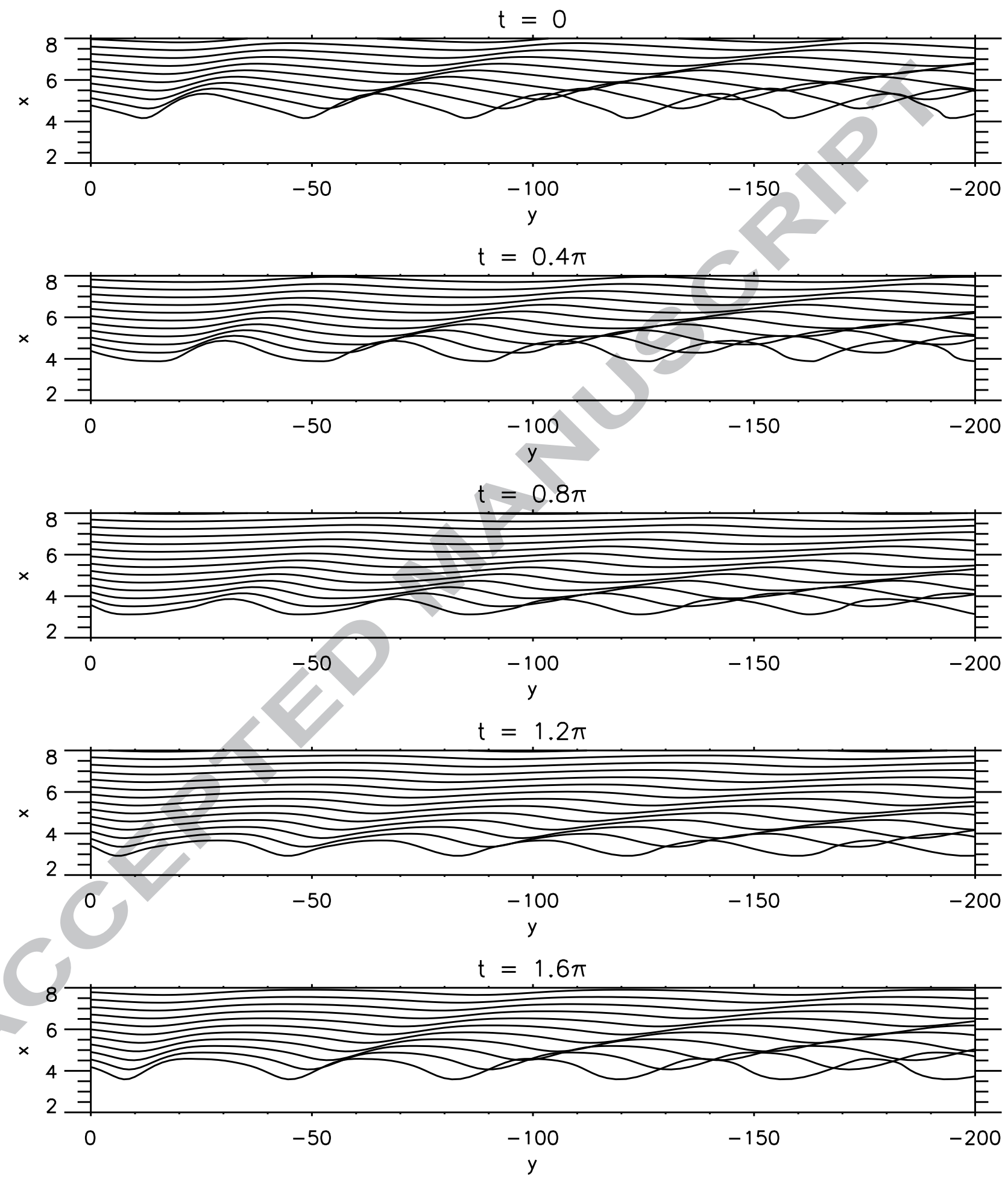

Fig. 5.- Family of streamlines for different times $\mathrm{t}$ for the parameters $e_{m}=0.75, \tau_{m}=0$, $e_{p}=0$ and $\tau_{p}=0$. 


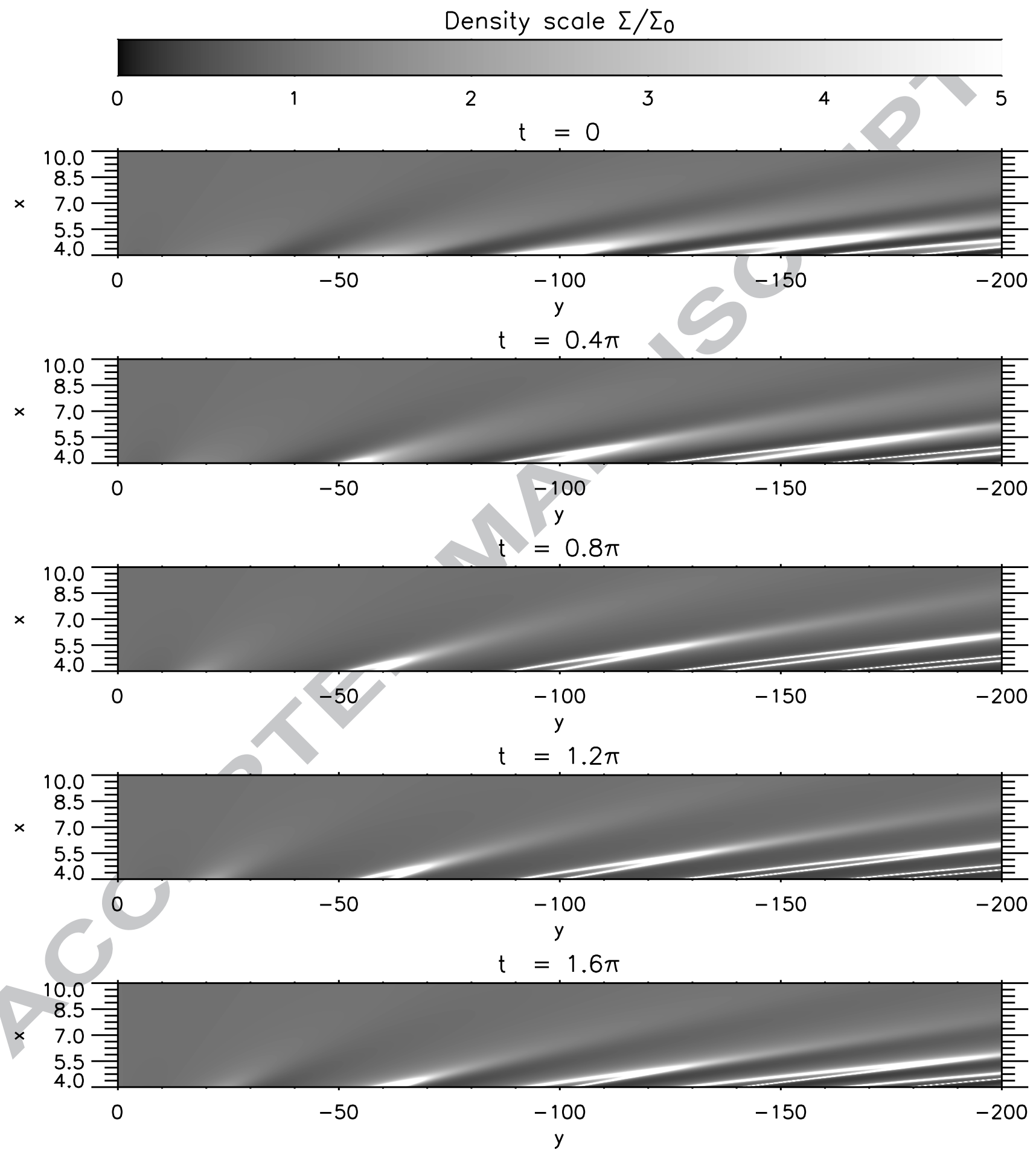

Fig. 6.- Wake density plot at different times $t$ for the parameters $e_{m}=0.5$ and $\tau=0$. Terms of the order $X^{-5}$ and larger have been chosen from the analytical solution for calculating the density. 

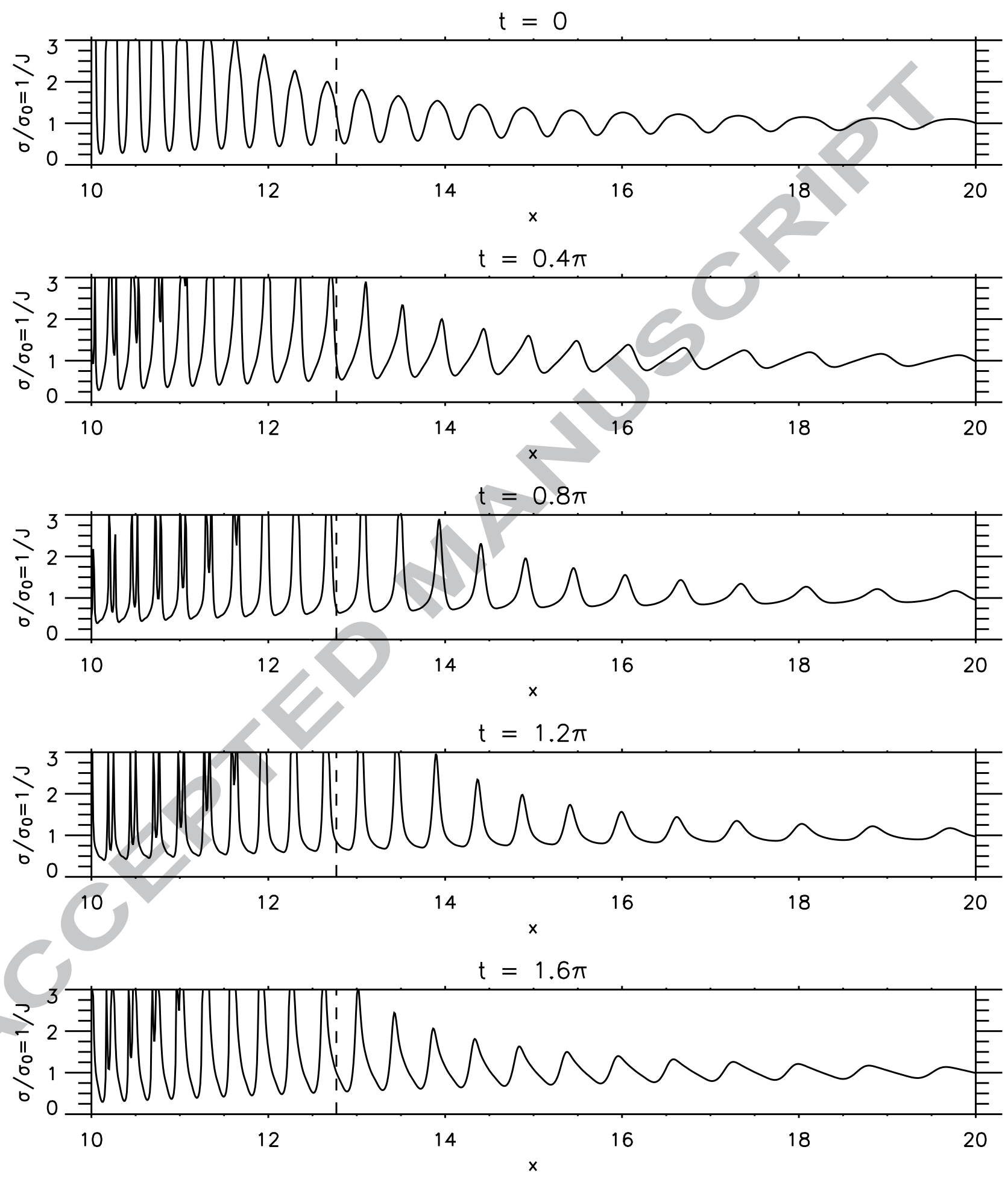

Fig. 7.- Radial wake density profiles at different times $t$ for the parameters $y=-4000$, $e_{m}=1.5$ and $\tau=0$. The density has been calculated from the analytical model equation (53) including terms of the order $X^{-5}$ and larger. The dashed line represents an estimate for the region where streamline crossing can appear, and the nonlinearity parameter becomes unity. The streamlines do not cross outside from this position. 
Density scale $\Sigma / \Sigma_{0}$

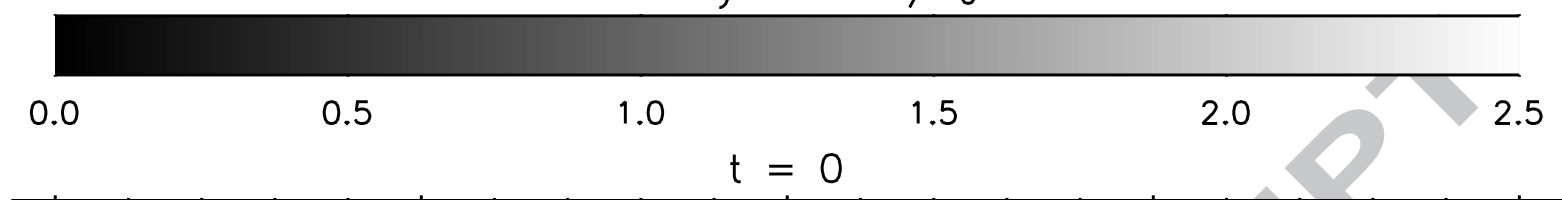
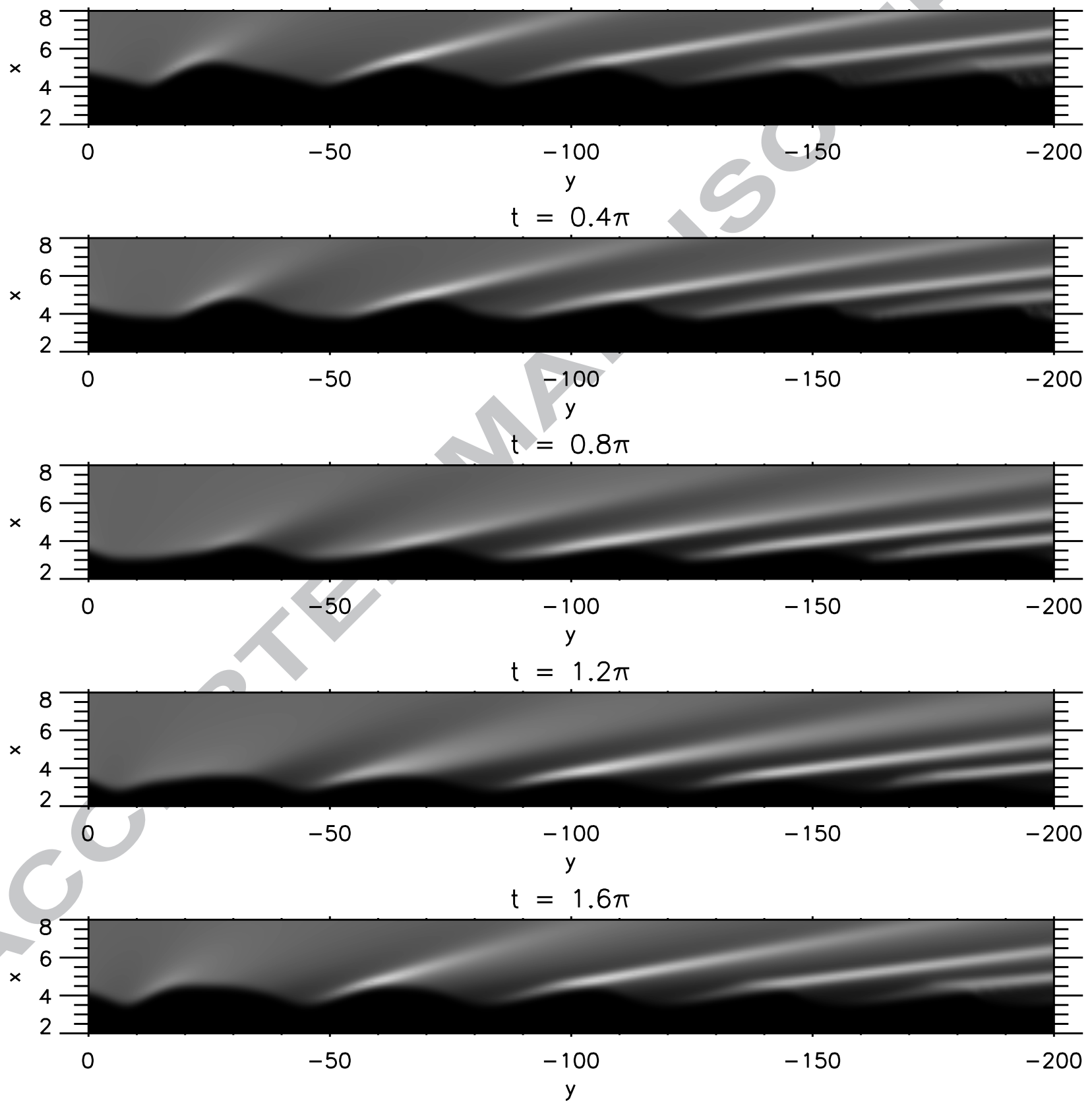

Fig. 8.- Wake density plot at different times $t$ for the same parameters as figure 5. The mean position of the gap edge is set to $X=4$, and the radial thermal velocity has been chosen to $c_{x}=0.2$. 

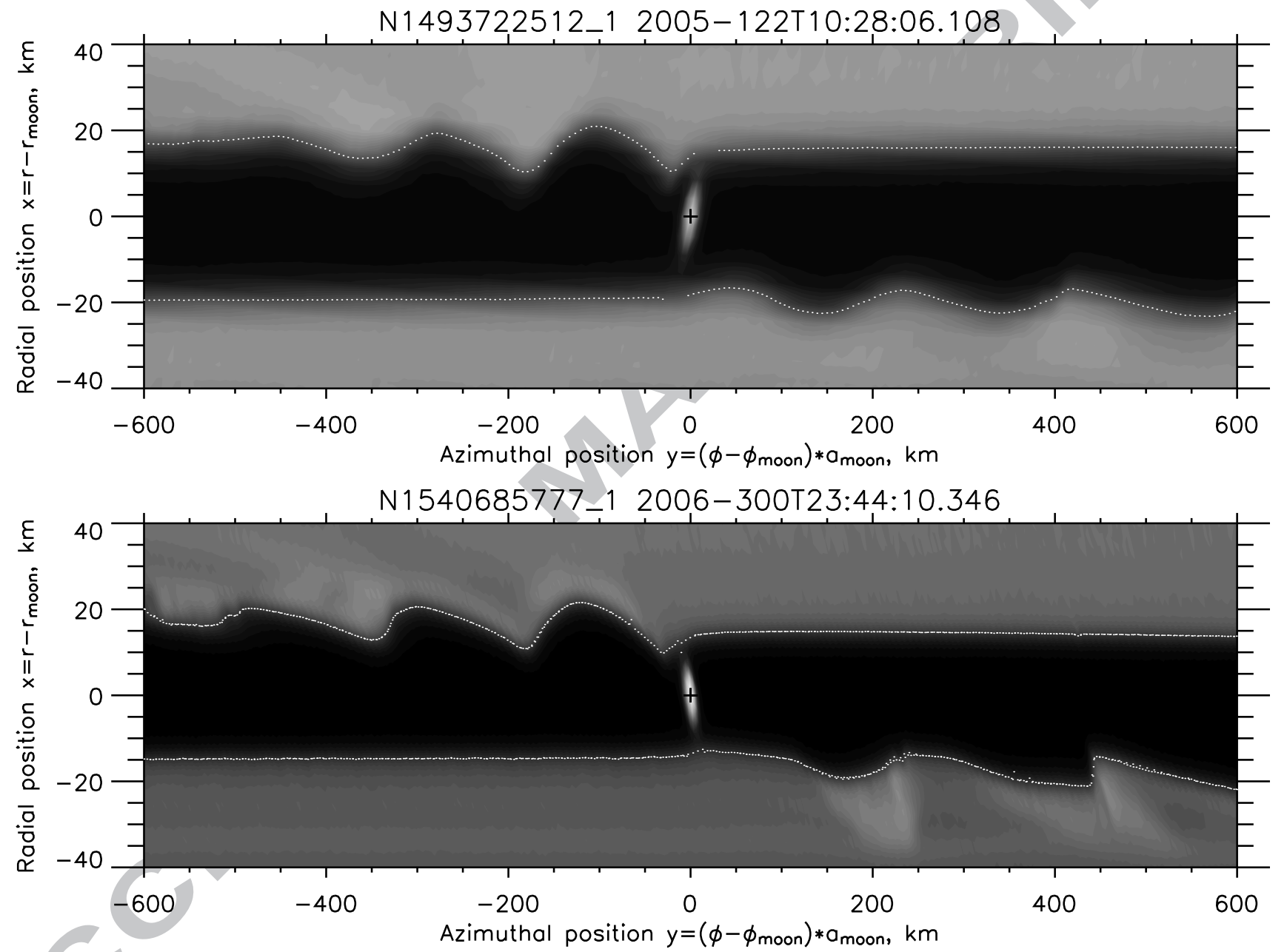

Fig. 9.- Plot of the moon Daphnis and of the Keeler gap in the vicinity of the moon. The center of the moon is marked by a cross. The dotted lines represent the fits of the gap edges. 

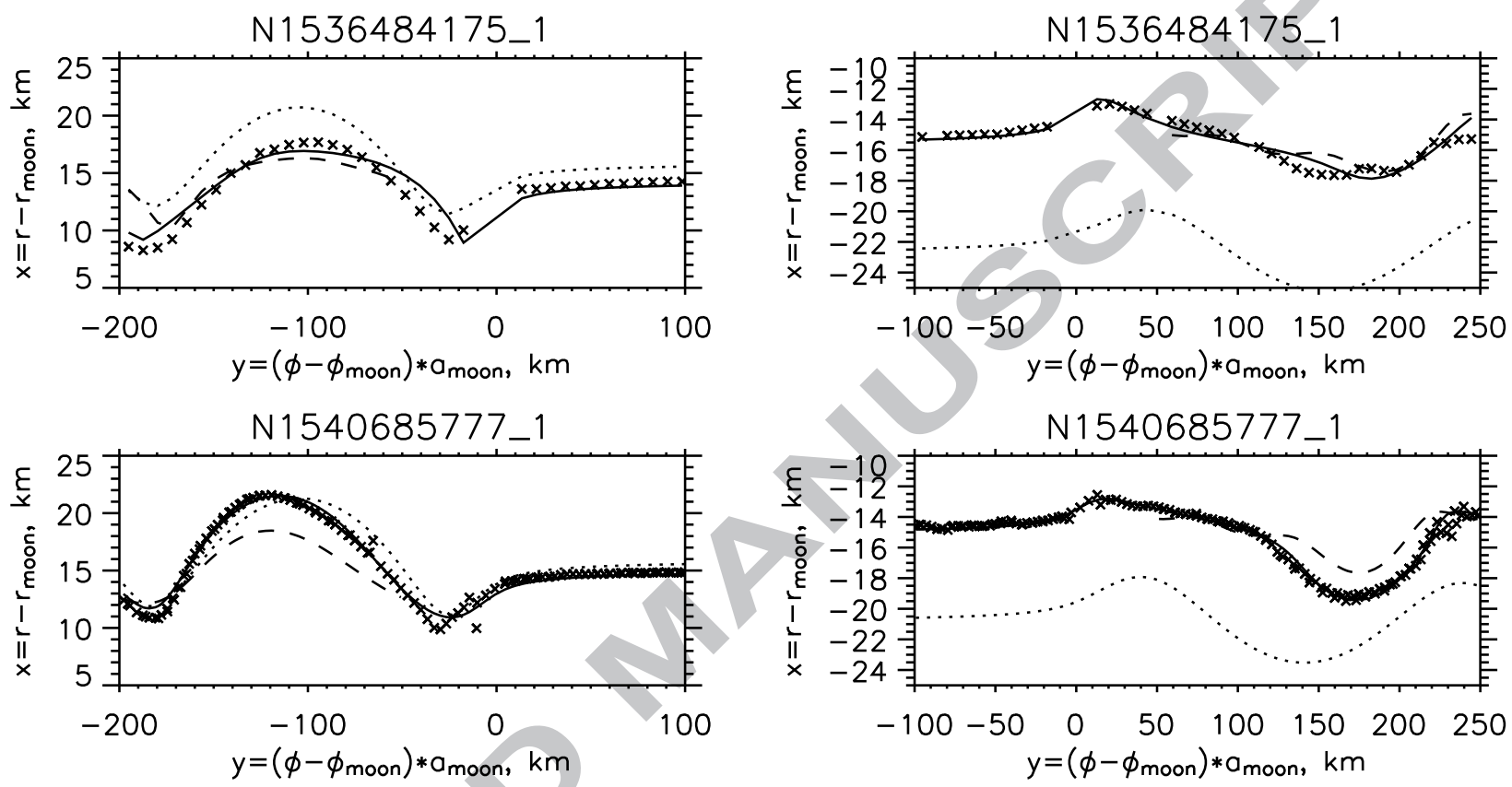

Fig. 10.- Comparison between measured edge positions (crosses) and fitted numerical streamline solution (solid line, see section 5). The results for outer and inner edge are shown in the left and the right column, respectively. The deviations are much smaller than the alternative numerical solution for initially circular orbits (dotted lines), but not negligible. The dashed line represents the 4th order analytical solution (eqns. (47)-(48) and (C1)(C2)), which deviates significantly, especially in the case of the outer edge because of the small impact parameter. Nevertheless, in case of the inner edge the analytical model provides a much better representation of the edge, because the eccentricity of the edge is taken into account. 

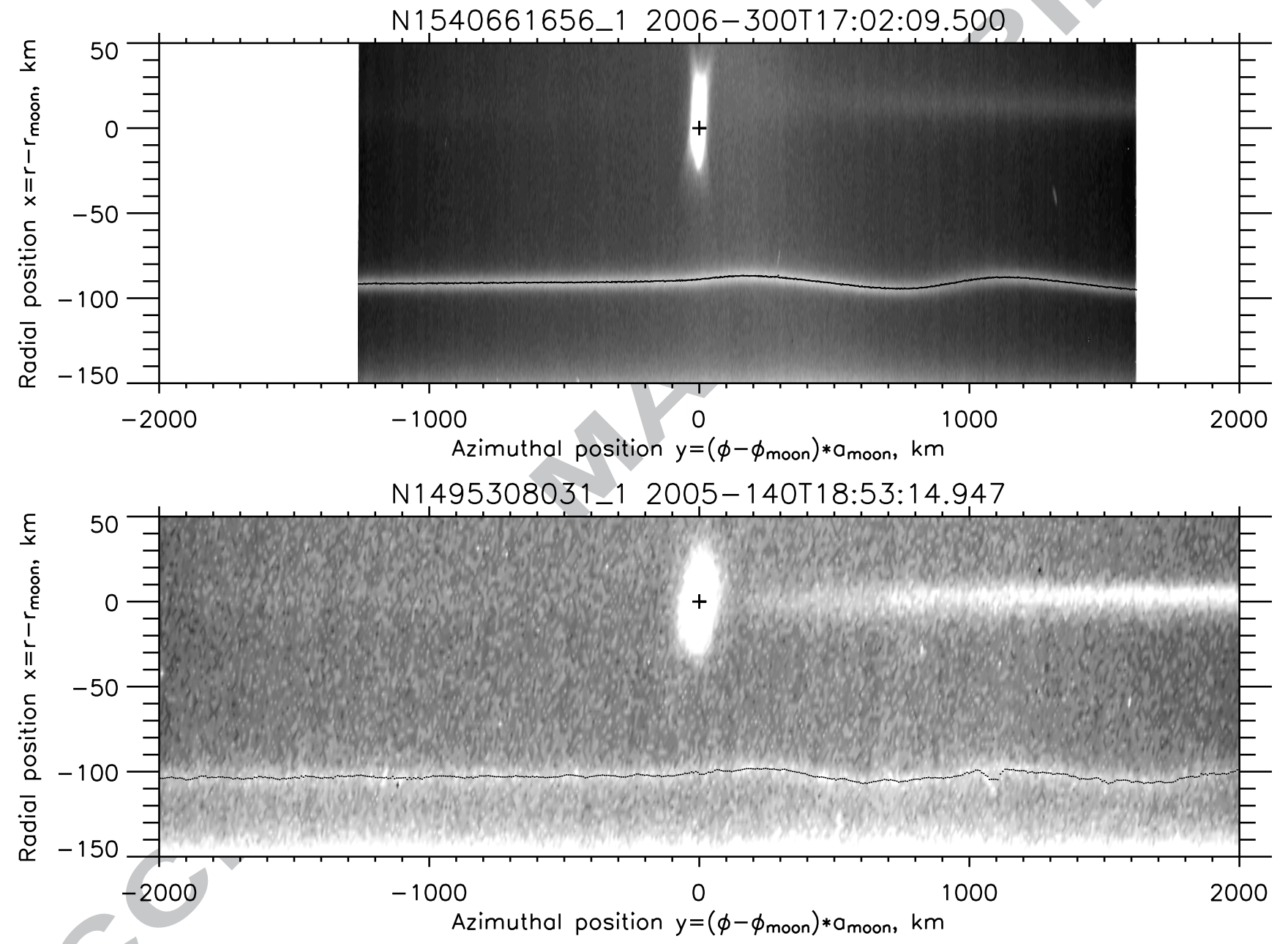

Fig. 11. - Pan and the inner Encke gap ringlet. The center of the moon is marked by a cross. The lines represent fits to the mean radial position of the ringlet in the Encke gap. 


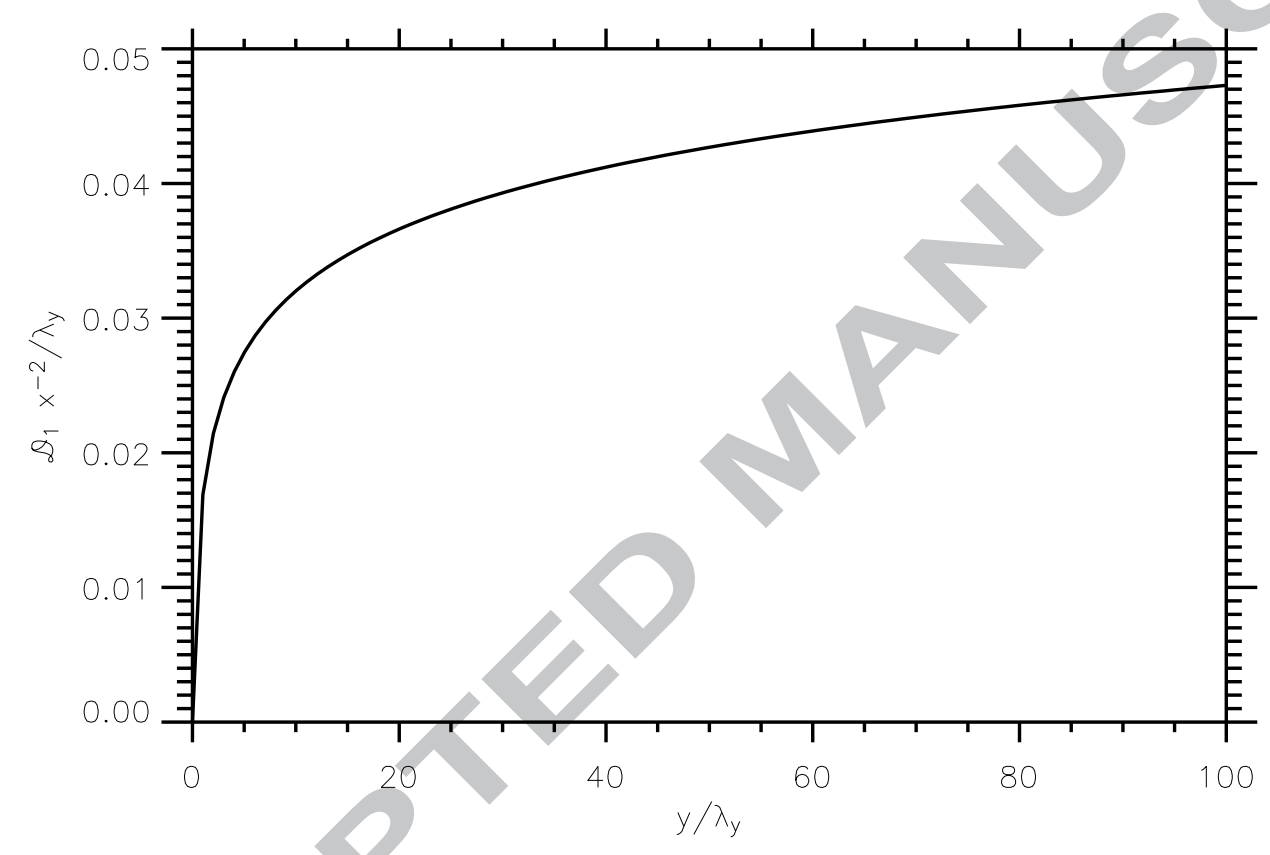

Fig. 12.- The azimuthal shift term $\mathcal{D}_{1}(y) x^{-2}$ plotted versus the azimuthal coordinate $y$. The term $\mathcal{D}_{1}(y) x^{-2}$ grows slowly and remains smaller than $5 \%$ of one wake cycle even after 100 wake cycles. 
Table 2: Parameters fitted from the outer Keeler gap edge

\begin{tabular}{cccccc}
\hline \hline Image name & Var, $\mathrm{km}^{2}$ & $h, \mathrm{~km}$ & $x_{0}$ & $e$ & $\tau-t+t_{\text {epoch }}$ \\
\hline $\mathrm{N} 1493722512$ & 0.35 & $5.06_{+0.13}^{-0.13}$ & $3.14_{+0.09}^{-0.10}$ & $0.23_{+0.16}^{-0.15}$ & $4.86_{+0.51}^{-0.44}$ \\
$\mathrm{~N} 1493722609$ & 0.28 & $5.09_{+0.11}^{-0.12}$ & $3.17_{+0.07}^{-0.07}$ & $0.23_{+0.13}^{-0.15}$ & $4.38_{+0.32}^{-0.38}$ \\
$\mathrm{~N} 1536484175$ & 0.94 & $4.87_{+0.24}^{-0.21}$ & $3.25_{+0.20}^{-0.17}$ & $0.63_{+0.26}^{-0.26}$ & $5.29_{+0.38}^{-0.32}$ \\
$\mathrm{~N} 1540685777$ & 0.24 & $5.01_{+0.08}^{-0.08}$ & $3.16_{+0.07}^{-0.07}$ & $0.24_{+0.08}^{-0.10}$ & $0.74_{+0.32}^{-0.38}$ \\
$\mathrm{~N} 1547691181$ & 0.68 & $5.06_{+0.17}^{-0.17}$ & $3.12_{+0.13}^{-0.12}$ & $0.01_{+0.13}^{-0.01}$ & $3.33_{+3.11}^{-3.11}$ \\
$\mathrm{~N} 1547691776$ & 0.27 & $4.94_{+0.11}^{-0.09}$ & $3.13_{+0.08}^{-0.08}$ & $0.02_{+0.12}^{-0.02}$ & $1.52_{+3.11}^{-3.11}$ \\
$\mathrm{~N} 1553466638$ & 0.75 & $5.03_{+0.16}^{-0.16}$ & $3.15_{+0.11}^{-0.11}$ & $0.15_{+0.23}^{-0.15}$ & $4.10_{+1.14}^{-1.33}$ \\
$\mathrm{~N} 1553469038$ & 1.05 & $4.68_{+0.21}^{-0.21}$ & $3.34_{+0.17}^{-0.14}$ & $0.34_{+0.17}^{-0.19}$ & $2.41_{+1.33}^{-0.63}$ \\
\hline
\end{tabular}

Note. — see Table 1

Table 3: Parameters fitted from the inner Encke gap ringlet

\begin{tabular}{cccccc}
\hline \hline Image name & Var, $\mathrm{km}$ & \multicolumn{1}{c}{$h, \mathrm{~km}$} & $x_{0}$ & $e$ & $\tau-t+t_{\text {epoch }}$ \\
\hline N1540661656 & 0.94 & $17.52_{+0.16}^{-0.14}$ & $-5.66_{+0.05}^{-0.04}$ & $0.49_{+0.04}^{-0.04}$ & $3.14_{+0.44}^{-0.44}$ \\
N1495308031 & 0.72 & $16.54_{+0.13}^{-0.13}$ & $-6.00_{+0.03}^{-0.03}$ & $0.28_{+0.06}^{-0.06}$ & $0.65_{+0.19}^{-1.52}$ \\
N1495308064 & 0.81 & $17.93_{+0.15}^{-0.15}$ & $-5.58_{+0.04}^{-0.02}$ & $0.31_{+0.09}^{-0.09}$ & $1.08_{+0.19}^{-2.22}$ \\
N1495308098 & 0.60 & $17.58_{+0.09}^{-0.11}$ & $-5.69_{+0.03}^{-0.04}$ & $0.21_{+0.05}^{-0.05}$ & $0.82_{+0.25}^{-1.84}$ \\
N1495308131 & 1.22 & $18.04_{+0.19}^{-0.16}$ & $-5.59_{+0.04}^{-0.05}$ & $0.27_{+0.18}^{-0.19}$ & $1.23_{+0.19}^{-2.67}$ \\
\hline
\end{tabular}

Note. - see Table 1 
Table 4: Numerical values of $\mathcal{A}_{i}, \mathcal{B}_{i}, \mathcal{C}_{i}$ and $\mathcal{D}_{i}$

\begin{tabular}{rrrrr}
\hline $\mathrm{i}$ & $\mathcal{A}_{i}$ & $\mathcal{B}_{i}$ & $\mathcal{C}_{i}$ & $\mathcal{D}_{i}$ \\
\hline 1 & -6.7187 & 0 & 0 & $\infty$ \\
2 & 0 & 8.7147 & -8.9583 & 0 \\
3 & 12.7147 & 0 & 0 & -4.8482 \\
4 & 15.5509 & 0 & 0 & -26.1440 \\
5 & 0 & -31.1017 & 28.5724 & 0 \\
6 & -16.6954 & 0 & 0 & 38.1440 \\
\hline
\end{tabular}


Table 5: Parameters of Keeler gap images

\begin{tabular}{|c|c|c|c|c|c|c|c|}
\hline Image & $\begin{array}{c}\text { Image time } \\
\text { (UTC) }\end{array}$ & $\begin{array}{c}\text { Exposure } \\
{[\mathrm{ms}]}\end{array}$ & $\begin{array}{c}\text { Radial } \\
\text { resolution } \\
{[\mathrm{km} / \text { pixel }]}\end{array}$ & $\begin{array}{l}\text { Azimuthal } \\
\text { resolution } \\
{[\mathrm{km} / \text { pixel }]}\end{array}$ & $\begin{array}{c}\text { Phase } \\
\text { angle } \\
\text { [degree] }\end{array}$ & $\begin{array}{c}\text { Incidence } \\
\text { angle } \\
\text { [degree] }\end{array}$ & $\begin{array}{c}\text { Emission } \\
\text { angle } \\
\text { [degree] }\end{array}$ \\
\hline N1493722512 & 2005-122T10:28:06.108 & 180.00 & 4.48 & 600 & 13.54 & 111.83 & 114.50 \\
\hline N1493722609 & 2005-122T10:29:43.108 & 180.00 & 4.44 & co & 13.67 & 111.83 & 114.45 \\
\hline N1536484175 & 2006-252Т08:37:55.129 & 820.00 & 2.36 & & 114.29 & 105.94 & 107.99 \\
\hline N1540685777 & 2006-300T23:44:10.346 & 260.00 & 3.02 & 3.94 & 35.89 & 105.28 & 115.19 \\
\hline N1547691181 & 2007-017T01:40:09.835 & 260.00 & -1 & 8.59 & 17.59 & 104.15 & 121.52 \\
\hline N1547691776 & 2007-017T01:50:04.831 & 260.00 & & 8.88 & 16.91 & 104.15 & 120.87 \\
\hline N1553466638 & 2007-083Т21:57:10.289 & 00 & 6.90 & 12.93 & 19.79 & 103.20 & 113.90 \\
\hline N1553469038 & 2007-083T22:37:10.274 & 120.00 & 5.36 & 13.14 & 20.78 & 103.20 & 114.08 \\
\hline
\end{tabular}


Table 6: Parameters of Encke gap images

\begin{tabular}{ccccccccc}
\hline \hline Image & $\begin{array}{c}\text { Image time } \\
\text { (UTC) }\end{array}$ & Exposure & $\begin{array}{c}\text { Radial } \\
\text { resolution }\end{array}$ & $\begin{array}{c}\text { Azimuthal } \\
\text { resolution }\end{array}$ & $\begin{array}{c}\text { Phase } \\
\text { angle }\end{array}$ & $\begin{array}{c}\text { Incidence } \\
\text { angle }\end{array}$ & $\begin{array}{c}\text { Emission } \\
\text { angle } \\
{[\text { degree] }}\end{array}$ \\
\hline N1540661656 & $2006-300 \mathrm{~T} 17: 02: 09.500$ & 1000.00 & 2.43 & 2.79 & 86.18 & 105.28 & 141.77 \\
N1495308031 & $2005-140 \mathrm{~km} 18: 53: 14.947$ & 18000.00 & 2.86 & 8.15 & 3.70 & 111.64 & 110.36 \\
N1495308064 & $2005-140 \mathrm{~T} 18: 53: 47.954$ & 380.00 & 2.87 & 8.16 & 3.68 & 111.64 & 110.33 \\
N1495308098 & $2005-140 \mathrm{~T} 18: 54: 21.958$ & 2000.00 & 2.88 & 8.17 & 3.66 & 111.64 & 110.31 \\
N1495308131 & $2005-140 \mathrm{~T} 18: 54: 54.962$ & 260.00 & 2.89 & 8.18 & 3.64 & 111.64 & 110.28 \\
\hline
\end{tabular}

